\title{
26. LATE QUATERNARY STABLE ISOTOPIC STRATIGRAPHY OF HOLE 910A, YERMAK PLATEAU, ARCTIC OCEAN: RELATIONS WITH SVALBARD/BARENTS SEA ICE SHEET HISTORY ${ }^{1}$
}

\author{
Benjamin P. Flower ${ }^{2}$
}

\begin{abstract}
Ocean Drilling Program Hole $910 \mathrm{~A}$ on the Yermak Plateau $\left(80^{\circ} \mathrm{N}, 5^{\circ} \mathrm{E}\right)$ at $552 \mathrm{~m}$ water depth provides one of the first opportunities for high-resolution studies of Quaternary Arctic Ocean climates. Oxygen and carbon isotopic data based on Neogloboquadrina pachyderma (sinistrally coiling) from the top $24.5 \mathrm{~m}$ at $10 \mathrm{~cm}$ sampling resolution provide both stratigraphic age control and a history of middle through late Quaternary surface water environments. The $\delta^{18} \mathrm{O}$ data reveal oxygen isotope Stages 1 through 16 (and possibly 17), with an amplitude of $\sim 1 \%$. Carbon isotopic values in the section from $\sim 19.5$ to $24.5 \mathrm{~m}$ below seafloor (mbsf) exhibit a series of $\sim 1 \%$ fluctuations, followed by a permanent $1 \%$ increase at $\sim 19.5 \mathrm{mbsf}$. Above $\sim 19.5$ mbsf, $\delta^{13} \mathrm{C}$ values are relatively stable, except for large spikes of $\sim 0.5 \%$, mainly during inferred interglacials. Significantly, the section from $\sim 19.5$ to $24.5 \mathrm{mbsf}$ is a remarkable "overconsolidated section," marked by high values for bulk density and sediment strength. The large variations in $\delta^{13} \mathrm{C}$ below $\sim 19.5 \mathrm{mbsf}$, and the overconsolidated nature of the section, suggest a fundamentally different sedimentary environment. Possible explanations include (1) episodic grounding of a marine-based ice sheet, perhaps derived from the Barents Sea ice sheet and buttressed by Svalbard, which overcompacted the sediment and strongly influenced surface water chemistry on the Yermak Plateau, and/or (2) coarser grained glacial sedimentation that allowed enhanced compaction during this interval. Oxygen isotopic stratigraphy suggests that overconsolidation ended near the Stage $17 / 16$ boundary ( $670 \mathrm{ka})$, reflecting a major change in state of the Svalbard/Barents Sea ice sheet.
\end{abstract}

\section{INTRODUCTION}

The Yermak Plateau is an aseismic topographic marginal high about $100 \mathrm{~km}$ north of Svalbard. The plateau is an Eocene (?) volcanic edifice, although basement has not been drilled and, consequently, the age is not well known. The Yermak Plateau lies just north of the Arctic Gateway at the Fram Strait, which connects the Arctic Ocean and the Norwegian-Greenland Sea. The Yermak Plateau is seasonally ice free in the present day, as a result of warm waters from the West Spitsbergen Current (WSC). This current is an extension of the warm Norwegian Current that splits south of Svalbard into a western branch (the WSC, flowing through the Fram Strait) and an eastern branch (flowing through the Barents Sea) into the Arctic Ocean.

Low sea-ice conditions in the summer of 1993 allowed the JOIDES Resolution to drill at three sites in a depth transect on the Yermak Plateau. Sites 910,911, and 912 were drilled at 552, 902, and $1037 \mathrm{~m}$ water depth (mwd), respectively, during Ocean Drilling Program (ODP) Leg 151 (Fig. 1). Sediments recovered at Site 910 $\left(80^{\circ} 16^{\prime} \mathrm{N}, 6^{\circ} 35^{\prime} \mathrm{E}\right)$ range in age from late Pliocene (in Hole $910 \mathrm{C}$ only) to Holocene; the total depth drilled was $506 \mathrm{~m}$ below seafloor (mbsf). The sediments are nearly homogeneous, dominantly finegrained silty clays, with a large ice-rafted debris (IRD) fraction. Carbonate material was sparse, partly because of dilution by terrestrially derived material. Dropstones were found throughout the sequence, attesting to a high flux of debris-laden icebergs (Myhre, Thiede, Firth, et al., 1995). Recovery in the Quaternary at Hole 910A was nearly $100 \%$ from 0 to $24.5 \mathrm{mbsf}$, with good magnetostratigraphic and biostratigraphic age control (Shipboard Scientific Party, 1995; Sato and Kameo, this volume).

Thiede, J., Myhre, A.M., Firth, J.V., Johnson, G.L., and Ruddiman, W.F. (Eds.), 1996. Proc. ODP, Sci. Results, 151: College Station, TX (Ocean Drilling Program).

Institute of Marine Sciences and Earth Sciences Board, University of California, Santa Cruz, Santa Cruz, CA 95064, U.S.A. flower@earthsci.ucsc.edu
Drilling in Hole 910A was inhibited by an "overconsolidated section" below $\sim 19.5$ mbsf, marked by large increases downcore in wetbulk density, from $\sim 1.3$ to $1.7 \mathrm{~g} / \mathrm{cm}^{3}$, and in sediment strength, from $\sim 150$ to $300 \mathrm{mPa}$ (Site 910; Shipboard Scientific Party, 1995). Sediment strength increased gradually from $\sim 16$ to 19 mbsf and rapidly

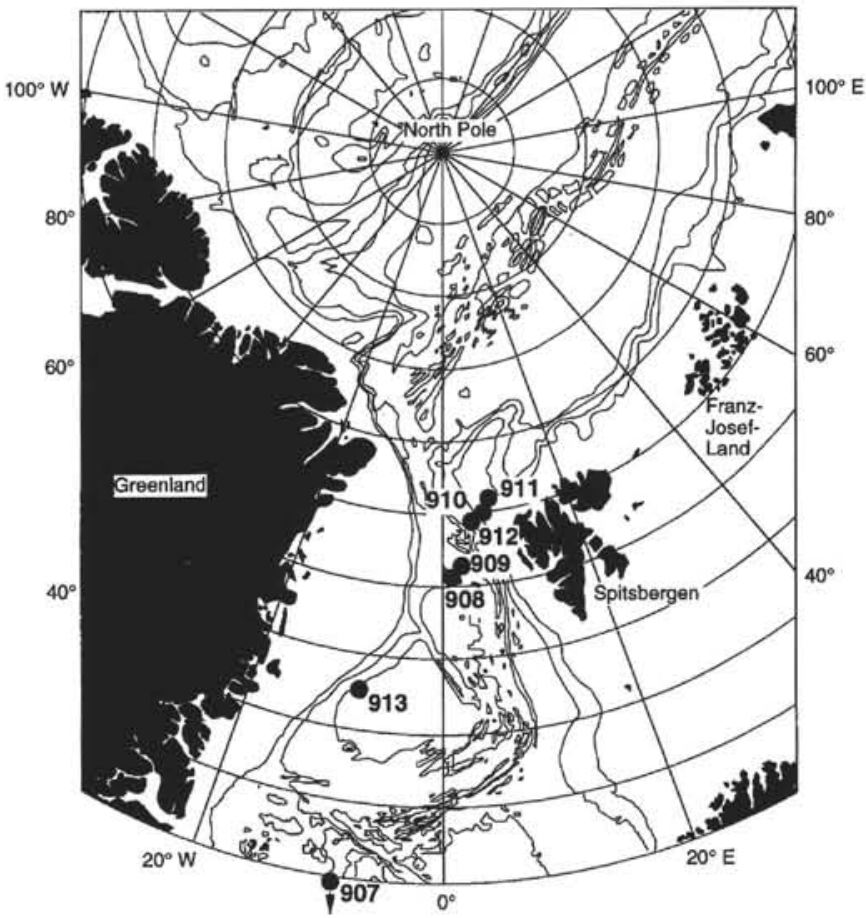

Figure 1. Location map of the Arctic Gateway region showing Leg 151 drill sites and important basins and ridges in the area. Sites 910,911, and 912 are on the Yermak Plateau at $\sim 80^{\circ} \mathrm{N}$. Bathymetric contour interval is $1000 \mathrm{~m}$. 
centered at 19.5 mbsf. On board ship, this section was classified as silty clays to clayey silts. The thickness of this section is not well known because of core recovery problems below $\sim 35 \mathrm{mbsf}$; the base is known to be between $\sim 70$ and 95 mbsf (Site 910; Shipboard Scientific Party, 1995). Only limited increases in consolidation were observed in the upper parts of Site 911 (902 mwd) and Site 912 (1037 mwd) on the Yermak Plateau. Also, Site 910 seismic stratigraphy revealed an acoustically chaotic upper section, whereas seismic sections at Sites 911 and 912 are well stratified (Myhre, Thiede, Firth, et al., 1995).

Interpretations by the Leg 151 Shipboard Scientific Party for the cause of the remarkable overconsolidated section at Site 910 include (1) grounding of a marine-based ice sheet, perhaps derived from the Barents Sea ice sheet and buttressed by Svalbard, and/or (2) coarsergrained glacial sedimentation that enhanced compaction (Leg 151 Shipboard Scientific Party, 1994; Myhre, Thiede, Firth, et al., 1995). The timing and cause of the overconsolidated section has important implications for Svalbard/Barents Sea ice sheet history. In this contribution, oxygen isotopic stratigraphy in Hole 910A is used to date the termination of overconsolidation near the stage $17 / 16$ boundary at $\sim 670 \mathrm{ka}$, suggesting a major change in Svalbard/Barents Sea ice sheet history at this time.

\section{METHODS}

Samples were dried and weighed, with a small subsample archived for additional work. Samples were then washed over a $63-\mu \mathrm{m}$ sieve with deionized water only, then dried and weighed again. The $>250-\mu \mathrm{m}$ fraction was also weighed. Individual planktonic foraminifers were handpicked from the $>150-\mu \mathrm{m}$ to $<250-\mu \mathrm{m}$ size fraction for isotopic analysis. The species used throughout the sequence was N. pachyderma (sinistrally coiling).

Approximately 20 specimens for isotopic analysis were sonicated in methanol to remove adhering particles, roasted under vacuum at $375^{\circ} \mathrm{C}$ to oxidize organic contaminants, and reacted in orthophosphoric acid at $90^{\circ} \mathrm{C}$. The evolved $\mathrm{CO}_{2}$ gas was then analyzed on-line using an isotope ratio mass spectrometer. Analyses were made either at the University of California, Santa Barbara, in the laboratory of James Kennett or in the laboratory of Christina Ravelo and James Zachos at the University of California, Santa Cruz. Measurements at UC Santa Barbara were made using a Finnigan/MAT 251 isotope ratio mass spectrometer equipped with an Autoprep Systems carbonate preparation device; those at UC Santa Cruz were made using a Fisons Prism isotope ratio mass spectrometer equipped with an automated preparation device.

Laboratory precision, which was run daily and was based on NBS-20 standards $\left(-4.14 \%\right.$ for $\delta^{18} \mathrm{O},-1.06 \%$ for $\left.\delta^{13} \mathrm{C}\right)$ at UC Santa Barbara and on NBS-19 standards $\left(-1.85 \%\right.$ for $\delta^{18} \mathrm{O}, 2.03 \%$ for $\delta^{13} \mathrm{C}$ ) at UC Santa Cruz, is $0.1 \%$ or better. No significant interlaboratory offsets were found, based on analyses of the NBS-19 and -20 standards. Replicate analyses on approximately $20 \%$ of the samples demonstrated a reproducibility of $\pm 0.14 \% \circ$ and $\pm 0.12 \%$ (mean standard deviation) for $\delta^{18} \mathrm{O}$ and $\delta^{13} \mathrm{C}$, respectively. No corrections were applied for offsets from isotopic equilibrium in $N$. pachyderma $(\mathrm{s})$ for either $\delta^{18} \mathrm{O}$ or $\delta^{13} \mathrm{C}$. All isotopic data are expressed using standard $\delta$ notation in per mil relative to the Peedee belemnite (PDB) carbonate standard.

\section{RESULTS}

Oxygen isotopic data based on N. pachyderma (s) (Table 1) from 0 to 15 mbsf exhibit a series of $\sim 1 \%$ cycles, with values generally ranging from $\sim 3.2 \%$ to $4.2 \%$ (Fig. 2). The mean $\delta^{18} \mathrm{O}$ values from 15 to $24.5 \mathrm{mbsf}$ are $\sim 0.3 \%$ lower, and generally range from $\sim 2.9 \%$ o to $3.8 \%$. Numerous low- $\delta^{18} \mathrm{O}$ events occur from 19.8 to $23.7 \mathrm{mbsf}$.
The carbon isotopic data from 0 to $19.5 \mathrm{mbsf}$ generally range from $-0.2 \%$ o to $-0.5 \%$, except for several spikes of $\sim 0.5 \%$ at $0.06,1.1,4.2$, 7.9, 9.6, 16.33 and 16.9 mbsf (Fig. 2). Also, the $\delta^{13} \mathrm{C}$ values for the top $50 \mathrm{~cm}$ are from -0.1 to $+0.4 \%$, significantly higher than the entire lower section. The $\delta^{13} \mathrm{C}$ values from 19.5 to $24.5 \mathrm{mbsf}$ exhibit large variations of over $1 \%$ (ranging from about $-0.5 \%$ to $-1.8 \%$ ) followed by a large permanent increase of $\sim 1.3 \%$ centered at $\sim 19.5$ mbsf. The $\delta^{13} \mathrm{C}$ increase at $\sim 19.5 \mathrm{mbsf}$ coincides with the termination of the overconsolidated section.

The percentage sand fraction (wt $\%>63 \mu \mathrm{m}$ ) and percentage very coarse sand (wt $\%>250 \mu \mathrm{m}$ ) remain relatively constant throughout the sequence ( $\sim 20 \%$ and $\sim 3 \%$, respectively; Table 2$)$. Notable exceptions are in the top $\sim 50 \mathrm{~cm}$, at 22.0 and $23.8 \mathrm{mbsf}$.

\section{DISCUSSION}

Stable isotopic data based on $N$. pachyderma (s) provide an isotopic stratigraphy for Hole $910 \mathrm{~A}$. Almost $100 \%$ core recovery allows generation of nearly continuous isotopic records. The oxygen isotopic data largely reflect the well-known variations in global ice volume during late Quaternary glacial-interglacial cycles (e.g., Shackleton and Opdyke, 1973; Imbrie et al., 1984). Thus, oxygen isotopic stratigraphy provides age control for the sequence, including the top of the overconsolidated section.

\section{Age Control}

The oxygen isotopic data clearly show isotope Stages 1 through 16 (and possibly 17), including numerous substages (Fig. 2). For example, three $\delta^{18} \mathrm{O}$ minima are seen during Stage 5, which are inferred to correspond to isotope Stages 5a, -c, and -e. Many of the isotope stages are readily assigned (e.g., Stages $1-5,11$, and 15). Also, the stage $12 / 11$ transition stands out as a large, rapid decrease in $\delta^{18} \mathrm{O}$, which distinguishes it among late Quaternary deglaciations (Shackleton and Opdyke, 1973; Imbrie et al., 1984). Interpretation of the isotope stratigraphy is aided by the last occurrence (LO) of Pseudoemiliania lacunosa at $\sim 9.7 \pm 0.75 \mathrm{mbsf}$ (Sato and Kameo, this volume), which is independently dated at $408 \mathrm{ka}$ in Stage 12 (Thierstein et al., 1977; Niitsuma et al., 1991).

An age vs. depth plot showing selected oxygen isotope stage boundaries (ages from Imbrie et al., 1984; Shackleton et al., 1990) and the $P$. lacunosa LO datum is shown in Figure 3. These stratigraphic datums allow calculation of ages and sedimentation rates for the section. Sedimentation rates average $\sim 1.9 \mathrm{~cm} / \mathrm{k} . \mathrm{y}$. for the $0-245$ $\mathrm{ka}$ interval and $\sim 3.3 \mathrm{~cm} / \mathrm{k} . \mathrm{y}$. for the $245-620 \mathrm{ka}$ interval.

The age of the section below $\sim 19$ mbsf is more problematic, as the oxygen isotope stratigraphy is more ambiguous. Normal magnetic polarity for the entire sequence suggests an age within the Brunhes Epoch. The problem is that extrapolating the sedimentation rate for the 245-620 ka interval would place the Brunhes/Matuyama boundary at $\sim 22.3 \mathrm{mbsf}$, but no reversed polarity sediments were recovered. The most likely explanation is that sedimentation rates were higher below $\sim 19 \mathrm{mbsf}$; rates of at least $4.5 \mathrm{~cm} / \mathrm{k}$.y would be required to place the Brunhes/Matuyama boundary at or below $24.2 \mathrm{mbsf}$.

An alternative interpretation calls for at least one hiatus between 19 and 24.5 mbsf, such that the normal polarity interval from $\sim 19.5$ to 24.5 mbsf would represent the lower Brunhes and the Jaramillo Chrons, with much of the upper Matuyama missing ( 780-990 ka). Normal polarity sediments in this interval are not assignable to the Olduvai Chron (1.77-1.95 Ma), based on calcareous nannofossil biostratigraphy (Sato and Kameo, this volume). In this view, the $\sim 0.3 \%$ lower $\delta^{18} \mathrm{O}$ values from $\sim 19$ to $24.5 \mathrm{mbsf}$ might reflect the wellknown lower mean $\delta^{18} \mathrm{O}$ values of the middle Quaternary prior to Stage $22(\sim 900 \mathrm{ka})$.

However, this alternative requires unreasonable changes in sedimentation rates, particularly during the short Jaramillo Chron (0.99- 
Table 1. Stable isotopic data from Hole 910A.

\begin{tabular}{|c|c|c|c|c|c|c|c|c|c|}
\hline $\begin{array}{l}\text { Core, section, } \\
\text { interval }(\mathrm{cm})\end{array}$ & $\begin{array}{l}\text { Depth } \\
\text { (mbsf) }\end{array}$ & $\begin{array}{l}\text { Age } \\
\text { (ka) }\end{array}$ & $\begin{array}{c}\delta^{18} \mathrm{O} \\
(\% \text { PDB })\end{array}$ & $\begin{array}{c}\delta^{13} \mathrm{C} \\
(\% \circ \mathrm{PDB})\end{array}$ & $\begin{array}{l}\text { Core, section, } \\
\text { interval (cm) }\end{array}$ & $\begin{array}{l}\text { Depth } \\
\text { (mbsf) }\end{array}$ & $\begin{array}{l}\text { Age } \\
\text { (ka) }\end{array}$ & $\begin{array}{c}\delta^{18} \mathrm{O} \\
(\% \circ \mathrm{PDB})\end{array}$ & $\begin{array}{c}\delta^{13} \mathrm{C} \\
(\% \circ \mathrm{PDB})\end{array}$ \\
\hline $151-910 \mathrm{~A}-$ & & & & & $2 \mathrm{H}-3,23-25^{\mathrm{a}}$ & 8.73 & 384.46 & 3.85 & -0.52 \\
\hline $1 \mathrm{H}-1,6-8^{\mathrm{a}}$ & 0.06 & 5.54 & 3.18 & 0.41 & $2 \mathrm{H}-3,30-32$ & 8.80 & 386.76 & 3.59 & -0.56 \\
\hline $1 \mathrm{H}-1,20-22^{\mathrm{a}}$ & 0.20 & 14.70 & 4.25 & -0.01 & $2 \mathrm{H}-3,40-42^{\mathrm{a}}$ & 8.90 & 390.06 & 3.67 & -0.46 \\
\hline $1 \mathrm{H}-1,20-22$ & 0.20 & 14.70 & 3.95 & -0.13 & $2 \mathrm{H}-3,40-42^{\mathrm{a}}$ & 8.90 & 390.06 & 3.81 & -0.36 \\
\hline IH-1, 30-32 & 0.30 & 18.55 & 4.22 & -0.07 & $2 \mathrm{H}-3,50-52$ & 9.00 & 393.35 & 3.80 & -0.46 \\
\hline $1 \mathrm{H}-1,50-52$ & 0.50 & 26.25 & 4.18 & -0.04 & $2 \mathrm{H}-3,60-62^{\mathrm{a}}$ & 9.10 & 396.65 & 3.47 & -0.52 \\
\hline $1 \mathrm{H}-1,80-82^{\mathrm{a}}$ & 0.80 & 37.81 & 3.90 & -0.47 & $2 \mathrm{H}-3,70-72$ & 9.20 & 399.94 & 3.55 & -0.34 \\
\hline $1 \mathrm{H}-1,90-92$ & 0.90 & 41.66 & 4.17 & -0.21 & $2 \mathrm{H}-3,83-85$ & 9.33 & 404.22 & 3.63 & -0.50 \\
\hline $1 \mathrm{H}-1,100-102^{\mathrm{a}}$ & 1.00 & 45.52 & 4.03 & -0.12 & $2 \mathrm{H}-3,90-92$ & 9.40 & 406.53 & 3.41 & -0.79 \\
\hline IH-1, 110-112 & 1.10 & 49.37 & 3.46 & -0.67 & $2 \mathrm{H}-3,100-102^{\mathrm{a}}$ & 9.50 & 409.82 & 3.10 & -1.02 \\
\hline $1 \mathrm{H}-1,120-122^{\mathrm{a}}$ & 1.20 & 53.22 & 3.64 & -0.42 & $2 \mathrm{H}-3,100-102$ & 9.50 & 409.82 & 3.42 & -1.54 \\
\hline $1 \mathrm{H}-1,130-132$ & 1.30 & 57.07 & 3.56 & -0.33 & $2 \mathrm{H}-3,110-112$ & 9.60 & 413.12 & 3.03 & -1.36 \\
\hline $1 \mathrm{H}-1,140-142^{\mathrm{a}}$ & 1.40 & 60.82 & 3.93 & -0.26 & $2 \mathrm{H}-3,120-122$ & 9.70 & 416.41 & 3.71 & -0.55 \\
\hline $1 \mathrm{H}-2,10-12$ & 1.60 & 68.08 & 4.01 & -0.14 & $2 \mathrm{H}-3,130-132$ & 9.80 & 419.71 & 3.73 & -0.41 \\
\hline $1 \mathrm{H}-2,20-22^{\mathrm{a}}$ & 1.70 & 71.71 & 3.75 & -0.26 & $2 \mathrm{H}-3,140-142^{\mathrm{a}}$ & 9.90 & 423.00 & 3.83 & -0.47 \\
\hline $1 \mathrm{H}-2,30-32$ & 1.80 & 75.34 & 4.00 & -0.15 & $2 \mathrm{H}-4,10-12$ & 10.10 & 427.81 & 3.82 & -0.47 \\
\hline $1 \mathrm{H}-2,40-42^{\mathrm{a}}$ & 1.90 & 78.97 & 3.58 & -0.28 & $2 \mathrm{H}-4,23-25^{a}$ & 10.23 & 430.94 & 3.92 & -0.44 \\
\hline $1 \mathrm{H}-2,40-42$ & 1.90 & 78.97 & 3.88 & -0.36 & $2 \mathrm{H}-4,30-32$ & 10.30 & 432.62 & 3.96 & -0.43 \\
\hline $1 \mathrm{H}-2,50-52$ & 2.00 & 82.61 & 4.04 & -0.23 & $2 \mathrm{H}-4,40-42^{a}$ & 10.40 & 435.02 & 4.15 & -0.35 \\
\hline $1 \mathrm{H}-2,60-62^{a}$ & 2.10 & 86.24 & 3.96 & -0.31 & $2 \mathrm{H}-4,50-52$ & 10.50 & 437.43 & 3.41 & -0.46 \\
\hline $1 \mathrm{H}-2,70-72$ & 2.20 & 89.87 & 3.86 & -0.18 & $2 \mathrm{H}-4,50-52$ & 10.50 & 437.43 & 3.42 & -0.92 \\
\hline $1 \mathrm{H}-2,80-82^{\mathrm{a}}$ & 2.30 & 93.50 & 3.23 & -0.46 & $2 \mathrm{H}-4,60-62^{\mathrm{a}}$ & 10.60 & 439.83 & 3.76 & -0.59 \\
\hline $1 \mathrm{H}-2,80-82$ & 2.30 & 93.50 & 3.71 & -0.51 & $2 \mathrm{H}-4,70-72$ & 10.70 & 442.24 & 3.80 & -0.55 \\
\hline $1 \mathrm{H}-2,90-92$ & 2.40 & 97.13 & 4.00 & -0.32 & $2 \mathrm{H}-4,82-84$ & 10.82 & 445.12 & 4.01 & -0.41 \\
\hline $1 \mathrm{H}-2,100-102^{\mathrm{a}}$ & 2.50 & 100.76 & 3.64 & -0.05 & $2 \mathrm{H}-4,90-92$ & 10.90 & 447.05 & 3.83 & -0.47 \\
\hline $1 \mathrm{H}-2,110-112$ & 2.60 & 104.39 & 3.93 & -0.16 & $2 \mathrm{H}-4,100-102^{\mathrm{a}}$ & 11.00 & 449.45 & 3.64 & -0.43 \\
\hline $1 \mathrm{H}-2,120-122^{\mathrm{a}}$ & 2.70 & 108.03 & 3.59 & -0.33 & $2 \mathrm{H}-4,110-112$ & 11.10 & 451.86 & 3.75 & -0.39 \\
\hline $1 \mathrm{H}-2,120-122$ & 2.70 & 108.03 & 3.81 & -0.39 & $2 \mathrm{H}-4,120-122$ & 11.20 & 454.26 & 3.87 & -0.51 \\
\hline $1 \mathrm{H}-2,130-132$ & 2.80 & 111.66 & 3.71 & -0.14 & $2 \mathrm{H}-4,130-132$ & 11.30 & 456.67 & 3.55 & -0.39 \\
\hline $1 \mathrm{H}-2,140-142^{3}$ & 2.90 & 115.29 & 3.28 & -0.55 & $2 \mathrm{H}-5,10-12$ & 11.60 & 463.88 & 3.49 & -0.41 \\
\hline $1 \mathrm{H}-3,10-12$ & 3.10 & 122.55 & 3.95 & -0.36 & $2 \mathrm{H}-5,23-25^{\mathrm{a}}$ & 11.73 & 467.01 & 3.84 & -0.33 \\
\hline $1 \mathrm{H}-3,20-22^{\mathrm{a}}$ & 3.20 & 126.18 & 3.37 & -0.49 & $2 \mathrm{H}-5,30-32$ & 11.80 & 468.69 & 3.70 & -0.50 \\
\hline $1 \mathrm{H}-3,20-22$ & 3.20 & 126.18 & 2.97 & -0.61 & $2 \mathrm{H}-5,40-42$ & 11.90 & 471.10 & 4.06 & -0.38 \\
\hline $1 \mathrm{H}-3,30-32$ & 3.30 & 132.33 & 4.06 & -0.27 & $2 \mathrm{H}-5,50-52$ & 12.00 & 473.50 & 3.71 & -0.46 \\
\hline $1 \mathrm{H}-3,40-42^{\mathrm{a}}$ & 3.40 & 141.00 & 3.71 & -0.41 & $2 \mathrm{H}-5,60-62^{\mathrm{a}}$ & 12.10 & 475.90 & 3.31 & -0.23 \\
\hline $1 \mathrm{H}-3,40-42^{\mathrm{a}}$ & 3.40 & 141.00 & 3.75 & -0.23 & $2 \mathrm{H}-5,60-62$ & 12.10 & 475.90 & 3.85 & -0.47 \\
\hline $1 \mathrm{H}-3,50-52$ & 3.50 & 149.67 & 3.78 & -0.25 & $2 \mathrm{H}-5,70-72$ & 12.20 & 478.31 & 3.15 & -0.77 \\
\hline $1 \mathrm{H}-3,60-62^{\mathrm{a}}$ & 3.60 & 158.33 & 3.77 & -0.29 & $2 \mathrm{H}-5,80-82$ & 12.30 & 480.71 & 3.94 & -0.38 \\
\hline $1 \mathrm{H}-3,70-72$ & 3.70 & 167.00 & 3.88 & -0.39 & $2 \mathrm{H}-5,80-82$ & 12.30 & 480.71 & 3.76 & -0.48 \\
\hline $1 \mathrm{H}-3,80-82^{\mathrm{a}}$ & 3.80 & 175.67 & 3.63 & -0.30 & $2 \mathrm{H}-5,90-92$ & 12.40 & 483.12 & 3.56 & -0.56 \\
\hline $1 \mathrm{H}-3,90-92$ & 3.90 & 184.33 & 3.80 & -0.33 & $2 \mathrm{H}-5,100-102^{\mathrm{a}}$ & 12.50 & 485.52 & 3.76 & -0.55 \\
\hline $1 \mathrm{H}-3,100-102^{\mathrm{A}}$ & 4.00 & 193.00 & 3.81 & -0.33 & $2 \mathrm{H}-5,110-112$ & 12.60 & 487.93 & 3.58 & -0.40 \\
\hline $1 \mathrm{H}-3,110-112$ & 4.10 & 201.67 & 3.46 & -0.60 & $2 \mathrm{H}-5,120-122$ & 12.70 & 490.33 & 3.62 & -0.36 \\
\hline $1 \mathrm{H}-3,120-122^{\mathrm{a}}$ & 4.20 & 210.33 & 2.96 & -1.31 & $2 \mathrm{H}-5,130-132$ & 12.80 & 492.74 & 3.52 & -0.51 \\
\hline $1 \mathrm{H}-3,120-122$ & 4.20 & 210.33 & 3.39 & -0.43 & $2 \mathrm{H}-5,140-142^{\mathrm{a}}$ & 12.90 & 495.14 & 3.93 & -0.36 \\
\hline $1 \mathrm{H}-3,130-132$ & 4.30 & 219.00 & 3.95 & -0.29 & $2 \mathrm{H}-6,10-12$ & 13.10 & 499.95 & 3.58 & -0.55 \\
\hline $1 \mathrm{H}-3,140-142^{\mathrm{a}}$ & 4.40 & 227.67 & 3.78 & -0.33 & $2 \mathrm{H}-6,23-25$ & 13.23 & 503.08 & 4.02 & -0.36 \\
\hline $1 \mathrm{H}-4,10-12$ & 4.60 & 245.00 & 3.88 & -0.38 & $2 \mathrm{H}-6,23-25$ & 13.23 & 503.08 & 3.94 & -0.46 \\
\hline $1 \mathrm{H}-4,20-22^{\mathrm{a}}$ & 4.70 & 248.42 & 4.12 & -0.25 & $2 \mathrm{H}-6,30-32$ & 13.30 & 504.76 & 3.53 & -0.44 \\
\hline $1 \mathrm{H}-4,30-32$ & 4.80 & 251.84 & 4.03 & -0.23 & $2 \mathrm{H}-6,40-42^{3}$ & 13.40 & 507.17 & 3.38 & -0.48 \\
\hline $1 \mathrm{H}-4,40-42^{\mathrm{a}}$ & 4.90 & 255.25 & 3.75 & -0.36 & $2 \mathrm{H}-6,50-52$ & 13.50 & 509.57 & 3.75 & -0.31 \\
\hline $1 \mathrm{H}-4,50-52$ & 5.00 & 258.67 & 3.83 & -0.32 & $2 \mathrm{H}-6,60-62^{\mathrm{a}}$ & 13.60 & 511.98 & 3.71 & -0.20 \\
\hline $1 \mathrm{H}-4,60-62^{\mathrm{a}}$ & 5.10 & 262.09 & 3.83 & -0.34 & $2 \mathrm{H}-6,70-72$ & 13.70 & 514.38 & 3.25 & -0.33 \\
\hline $1 \mathrm{H}-4,70-72$ & 5.30 & 268.93 & 3.95 & -0.22 & $2 \mathrm{H}-6,80-82$ & 13.80 & 516.79 & 3.56 & -0.19 \\
\hline 1H-CC, $10-12$ & 5.36 & 270.98 & 3.97 & -0.47 & $2 \mathrm{H}-6,100-102^{\mathrm{a}}$ & 14.00 & 521.60 & 3.45 & -0.42 \\
\hline $1 \mathrm{H}-\mathrm{CC}, 18-20$ & 5.44 & 273.71 & 3.74 & -0.39 & $2 \mathrm{H}-\mathrm{CC}, 10-12$ & 14.20 & 527.15 & 3.86 & -0.33 \\
\hline $2 \mathrm{H}-1,10-12$ & 5.60 & 279.18 & 3.90 & -0.37 & $2 \mathrm{H}-\mathrm{CC}, 23-25^{\mathrm{a}}$ & 14.32 & 530.92 & 3.71 & -0.43 \\
\hline $2 \mathrm{H}-1,23-25^{\mathrm{a}}$ & 5.73 & 283.63 & 3.76 & -0.32 & $2 \mathrm{H}-\mathrm{CC}, 30-32$ & 14.40 & 533.44 & 3.91 & -0.47 \\
\hline $2 \mathrm{H}-1,30-32$ & 5.80 & 286.02 & 3.98 & -0.37 & $3 \mathrm{H}-1,10-12$ & 15.10 & 555.48 & 3.99 & -0.38 \\
\hline $2 \mathrm{H}-1,40-42$ & 5.90 & 289.44 & 4.04 & -0.22 & $3 \mathrm{H}-1,20-22^{\mathrm{a}}$ & 15.20 & 558.62 & 3.79 & -0.44 \\
\hline $2 \mathrm{H}-1,50-52$ & 6.00 & 292.85 & 4.11 & -0.29 & $3 \mathrm{H}-1,20-22^{\mathrm{a}}$ & 15.20 & 558.62 & 3.18 & -0.48 \\
\hline $2 \mathrm{H}-1,60-62^{\mathrm{a}}$ & 6.10 & 296.27 & 3.24 & -0.49 & $3 \mathrm{H}-1,30-32$ & 15.30 & 561.77 & 3.63 & -0.45 \\
\hline $2 \mathrm{H}-1,60-62$ & 6.10 & 296.27 & 3.47 & -0.25 & $3 \mathrm{H}-1,40-42$ & 15.40 & 564.92 & 3.77 & -0.36 \\
\hline $2 \mathrm{H}-1,70-72$ & 6.20 & 299.69 & 3.92 & -0.32 & $3 \mathrm{H}-1,50-52$ & 15.50 & 568.07 & 3.09 & -0.46 \\
\hline $2 \mathrm{H}-1,80-82^{\mathrm{a}}$ & 6.30 & 303.11 & 3.88 & -0.31 & $3 \mathrm{H}-1,60-62^{\mathrm{a}}$ & 15.60 & 571.21 & 3.26 & -0.51 \\
\hline $2 \mathrm{H}-1,90-92$ & 6.40 & 306.53 & 3.65 & -0.23 & $3 \mathrm{H}-1,70-72$ & 15.70 & 574.36 & 3.30 & -0.56 \\
\hline $2 \mathrm{H}-1,100-102^{\mathrm{a}}$ & 6.50 & 309.95 & 3.06 & -0.40 & $3 \mathrm{H}-1,81-83$ & 15.81 & 577.82 & 3.54 & -0.46 \\
\hline $2 \mathrm{H}-1,100-102^{\mathrm{a}}$ & 6.50 & 309.95 & 3.66 & -0.41 & $3 \mathrm{H}-1,90-92$ & 15.90 & 580.66 & 3.67 & -0.59 \\
\hline $2 \mathrm{H}-1,110-112$ & 6.60 & 313.36 & 3.49 & -0.51 & $3 \mathrm{H}-1,100-102^{\mathrm{a}}$ & 16.00 & 583.80 & 3.60 & -0.45 \\
\hline $2 \mathrm{H}-1,120-122$ & 6.70 & 316.78 & 4.04 & -0.35 & $3 \mathrm{H}-1,110-112$ & 16.10 & 586.95 & 3.74 & -0.30 \\
\hline $2 \mathrm{H}-1,120-122$ & 6.70 & 316.78 & 3.88 & -0.46 & $3 \mathrm{H}-1,120-122$ & 16.20 & 590.10 & 3.71 & -0.30 \\
\hline $2 \mathrm{H}-1,140-142^{\mathrm{a}}$ & 6.90 & 323.62 & 3.42 & -0.30 & $3 \mathrm{H}-1,133-135$ & 16.33 & 594.19 & 3.30 & -1.21 \\
\hline $2 \mathrm{H}-2,10-12$ & 7.10 & 330.45 & 3.68 & -0.35 & $3 \mathrm{H}-1,133-135$ & 16.33 & 594.19 & 3.16 & -0.80 \\
\hline $2 \mathrm{H}-2,23-35^{a}$ & 7.23 & 334.90 & 3.82 & -0.28 & $3 \mathrm{H}-1,140-142^{\mathrm{a}}$ & 16.40 & 596.39 & 3.13 & -0.66 \\
\hline $2 \mathrm{H}-2,30-32$ & 7.30 & 337.29 & 3.50 & -0.39 & $3 \mathrm{H}-2,30-32$ & 16.80 & 608.98 & 3.88 & -0.36 \\
\hline $2 \mathrm{H}-2,40-42^{a}$ & 7.40 & 340.65 & 4.09 & -0.27 & $3 \mathrm{H}-2,30-32$ & 16.80 & 608.98 & 3.03 & -1.96 \\
\hline $2 \mathrm{H}-2,50-52$ & 7.50 & 343.94 & 3.98 & -0.36 & $3 \mathrm{H}-2,40-42^{\mathrm{a}}$ & 16.90 & 612.13 & 2.73 & -1.21 \\
\hline $2 \mathrm{H}-2,60-62^{a}$ & 7.60 & 347.24 & 3.63 & -0.35 & $3 \mathrm{H}-2,40-42$ & 16.90 & 612.13 & 3.14 & -0.72 \\
\hline $2 \mathrm{H}-2,70-72$ & 7.70 & 350.53 & 3.54 & -0.20 & $3 \mathrm{H}-2,40-42$ & 16.90 & 612.13 & 3.50 & -1.31 \\
\hline $2 \mathrm{H}-2,80-82$ & 7.80 & 353.82 & 3.73 & -0.36 & $3 \mathrm{H}-2,50-52$ & 17.00 & 615.28 & 2.99 & -0.60 \\
\hline $2 \mathrm{H}-2,90-92$ & 7.90 & 357.12 & 3.55 & -1.11 & $3 \mathrm{H}-2,60-62^{\mathrm{a}}$ & 17.10 & 618.43 & 3.26 & -0.54 \\
\hline $2 \mathrm{H}-2,90-92$ & 7.90 & 357.12 & 4.00 & -3.12 & $3 \mathrm{H}-2,60-62$ & 17.10 & 618.43 & 3.59 & -0.48 \\
\hline $2 \mathrm{H}-2,100-102^{\mathrm{a}}$ & 8.00 & 360.41 & 3.66 & -0.30 & $3 \mathrm{H}-2,70-72$ & 17.20 & 621.14 & 3.41 & -0.29 \\
\hline $2 \mathrm{H}-2,110-112$ & 8.10 & 363.71 & 3.57 & -0.41 & $3 \mathrm{H}-2,81-83$ & 17.31 & 623.66 & 3.56 & -0.37 \\
\hline $2 \mathrm{H}-2,120-122$ & 8.20 & 367.00 & 3.94 & -0.32 & $3 \mathrm{H}-2,90-92$ & 17.40 & 625.72 & 3.73 & -0.51 \\
\hline $2 \mathrm{H}-2,130-132$ & 8.30 & 370.29 & 3.88 & -0.28 & $3 \mathrm{H}-2,100-102^{\mathrm{a}}$ & 17.50 & 672.8 & 3.51 & -0.48 \\
\hline $2 \mathrm{H}-2,140-142^{2}$ & 8.40 & 373.59 & 3.95 & -0.37 & $3 \mathrm{H}-2,100-102^{\mathrm{a}}$ & 17.50 & 628.01 & 3.24 & -0.53 \\
\hline $2 \mathrm{H}-3,10-12$ & 8.60 & 380.18 & 3.64 & -0.19 & $3 \mathrm{H}-2,110-112$ & 17.60 & 630.30 & 3.50 & -0.46 \\
\hline
\end{tabular}


Table 1 (continued).

\begin{tabular}{|c|c|c|c|c|}
\hline $\begin{array}{l}\text { Core, section, } \\
\text { interval }(\mathrm{cm})\end{array}$ & $\begin{array}{l}\text { Depth } \\
\text { (mbsf) }\end{array}$ & $\begin{array}{l}\text { Age } \\
(\mathrm{ka})\end{array}$ & $\begin{array}{c}\delta^{18} \mathrm{O} \\
(\% \circ \mathrm{PDB})\end{array}$ & $\begin{array}{c}\delta^{13} \mathrm{C} \\
(\% \circ \mathrm{PDB})\end{array}$ \\
\hline $3 \mathrm{H}-2,120-122$ & 17.70 & 632.58 & 3.49 & -0.45 \\
\hline $3 \mathrm{H}-2,130-132$ & 17.80 & 634.87 & 3.40 & -0.53 \\
\hline $3 \mathrm{H}-2,140-142^{\mathrm{a}}$ & 17.90 & 637.16 & 3,24 & -0.53 \\
\hline $3 \mathrm{H}-2,140-142$ & 17.90 & 637.16 & 3.62 & -0.50 \\
\hline $3 \mathrm{H}-3,10-12$ & 18.10 & 641.74 & 3.39 & -0.38 \\
\hline $3 \mathrm{H}-3,20-22$ & 18.20 & 644.02 & 3.59 & -0.73 \\
\hline $3 \mathrm{H}-3,30-32$ & 18.30 & 646.31 & 3.78 & -0.42 \\
\hline $3 \mathrm{H}-3,40-42^{\mathrm{a}}$ & 18.40 & 648.60 & 3.56 & -0.42 \\
\hline $3 \mathrm{H}-3,48-58$ & 18.48 & 650.43 & 3.77 & -0.58 \\
\hline $3 \mathrm{H}-3,60-62$ & 18.60 & 653.18 & 3.36 & -0.70 \\
\hline $3 \mathrm{H}-3,70-72$ & 18.70 & 655.46 & 3.58 & -0.55 \\
\hline $3 \mathrm{H}-3,80-82$ & 18.80 & 657.75 & 3.68 & -0.65 \\
\hline $3 \mathrm{H}-3,90-92$ & 18.90 & 660.04 & 3.71 & -0.35 \\
\hline $4 \mathrm{H}-1,10-12$ & 19.60 & 676.06 & 3.10 & -1.14 \\
\hline $4 \mathrm{H}-1,20-22^{\mathrm{a}}$ & 19.70 & 678.34 & 3.05 & -0.91 \\
\hline $4 \mathrm{H}-1,30-32$ & 19.80 & 680.63 & 2.90 & -1.09 \\
\hline $4 \mathrm{H}-1,40-42$ & 19.90 & 682.92 & 3.38 & -1.03 \\
\hline $4 \mathrm{H}-1,50-52$ & 20.00 & 685.21 & 3.64 & -0.86 \\
\hline $4 \mathrm{H}-1,60-62^{\mathrm{a}}$ & 20.10 & 687.50 & 3.04 & -1.26 \\
\hline $4 \mathrm{H}-1,70-72$ & 20.20 & 689.78 & 2.94 & -1.69 \\
\hline $4 \mathrm{H}-1,80-82$ & 20.30 & 692.07 & 2.98 & -1.15 \\
\hline $4 \mathrm{H}-1,90-92$ & 20.40 & 694.36 & 3.44 & -1.27 \\
\hline $4 \mathrm{H}-1,100-102^{\mathrm{a}}$ & 20.50 & 696.65 & 3.22 & -1.07 \\
\hline $4 \mathrm{H}-1,110-112$ & 20.60 & 698.94 & 3.73 & -0.93 \\
\hline $4 \mathrm{H}-1,110-112$ & 20.60 & 698.94 & 3.75 & -0.46 \\
\hline $4 \mathrm{H}-1,120-122$ & 20.70 & 701.22 & 3.27 & -1.41 \\
\hline $4 \mathrm{H}-1,130-132$ & 20.80 & 703.51 & 3.40 & -1.22 \\
\hline $4 \mathrm{H}-1,140-142^{a}$ & 20.90 & 705.80 & 2.97 & -1.84 \\
\hline $4 \mathrm{H}-1,140-142$ & 20.90 & 705.80 & 4.04 & -0.17 \\
\hline $4 \mathrm{H}-2,10-12$ & 21.10 & 710.38 & 3.30 & -0.96 \\
\hline $4 \mathrm{H}-2,18-20^{\mathrm{a}}$ & 21.18 & 712.21 & 3.00 & -0.90 \\
\hline $4 \mathrm{H}-2,30-32$ & 21.30 & 714.95 & 3.62 & -0.68 \\
\hline $4 \mathrm{H}-2,40-42^{\mathrm{a}}$ & 21.40 & 717.24 & 3.42 & -0.88 \\
\hline $4 \mathrm{H}-2,50-52$ & 21.50 & 719.53 & 3.57 & -0.91 \\
\hline $4 \mathrm{H}-2,60-62^{3}$ & 21.60 & 721.82 & 3.37 & -0.63 \\
\hline $4 \mathrm{H}-2,70-72$ & 21.70 & 724.10 & 3.71 & -0.66 \\
\hline $4 \mathrm{H}-2,80-82^{\mathrm{a}}$ & 21.80 & 726.39 & 3.76 & -0.65 \\
\hline $4 \mathrm{H}-2,90-92$ & 21.90 & 728.68 & 3.04 & -0.53 \\
\hline $4 \mathrm{H}-2,110-112$ & 22.10 & 733.26 & 4.52 & -0.40 \\
\hline $4 \mathrm{H}-2,110-112$ & 22.10 & 733.26 & 4.11 & -1.55 \\
\hline $4 \mathrm{H}-2,120-122$ & 22.20 & 735.54 & 3.30 & -1.20 \\
\hline $4 \mathrm{H}-2,130-132$ & 22.30 & 737.83 & 3.16 & -1.77 \\
\hline $4 \mathrm{H}-2,138-140$ & 22.38 & 739.66 & 3.24 & -1.43 \\
\hline $4 \mathrm{H}-3,10-12$ & 22.60 & 744.70 & 3.65 & -1.32 \\
\hline $4 \mathrm{H}-3,20-22^{\mathrm{a}}$ & 22.70 & 746.98 & 2.79 & -1.31 \\
\hline $4 \mathrm{H}-3,20-22$ & 22.70 & 746.98 & 3.22 & -0.99 \\
\hline $4 \mathrm{H}-3,30-32$ & 22.80 & 749.27 & 3.16 & -1.32 \\
\hline $4 \mathrm{H}-3,40-42$ & 22.90 & 751.56 & 3.54 & -1.40 \\
\hline $4 \mathrm{H}-3,50-52$ & 23.00 & 753.85 & 3.51 & -1.31 \\
\hline $4 \mathrm{H}-3,60-62^{\mathrm{a}}$ & 23.10 & 756.14 & 3.08 & -1.13 \\
\hline $4 \mathrm{H}-3,70-72$ & 23.20 & 758.42 & 3.38 & -1.21 \\
\hline $4 \mathrm{H}-3,80-82$ & 23.30 & 760.71 & 3.19 & -1.45 \\
\hline $4 \mathrm{H}-3,90-92$ & 23.40 & 763.00 & 3.40 & -1.16 \\
\hline $4 \mathrm{H}-3,100-102^{a}$ & 23.50 & 765.29 & 3.74 & -0.40 \\
\hline $4 \mathrm{H}-3,110-112$ & 23.60 & 767.58 & 3.17 & -1.32 \\
\hline $4 \mathrm{H}-3,120-122$ & 23.70 & 769.86 & 2.64 & -1.08 \\
\hline $4 \mathrm{H}-3,120-122$ & 23.70 & 769.86 & 3.24 & -1.38 \\
\hline $4 \mathrm{H}-4,10-12$ & 24.10 & 779.02 & 3.10 & -1.03 \\
\hline $4 \mathrm{H}-4,20-22^{3}$ & 24.20 & 781.78 & 3.68 & -0.71 \\
\hline $4 \mathrm{H}-4,20-22$ & 24.20 & 781.78 & 3.90 & -0.41 \\
\hline
\end{tabular}

Note: asample analyzed at the University of California, Santa Barbara. All other samples analyzed at the University of California, Santa Cruz.

1.07 Ma). Further, interpolation between the 15/16 stage boundary and the first occurrence (FO) of Gephyrocapsa oceanica (1.70 Ma; Raffi et al., 1993) between Sections 151-910C-7R-CC and 8R-CC (Sato and Kameo, this volume) yields a minimum estimate of $\sim 4.0$ $\mathrm{cm} / \mathrm{k}$.y. for the 17-60 mbsf interval. Based on this evidence for higher average sedimentation rates in this interval, I concur with the assignment of Hole 910A to the Brunhes Chron (Shipboard Scientific Party, 1995) and conclude that no major hiatus exists in the upper $24.5 \mathrm{~m}$ in Hole $910 \mathrm{~A}$ and assign ages for the 19-24.5 mbsf section accordingly. As an aside, a hiatus that removed much of the lower Quaternary to upper Pliocene is found at the base of the overconsolidated section between $\sim 70$ and $95 \mathrm{mbsf}$, based on biostratigraphy (Site 910; Myhre, Thiede, Firth, et al., 1995). These interpretations await independent assessment of ages within the overconsolidated section, such as strontium isotopic stratigraphy.

Thus, oxygen isotopic stratigraphy in the overlying sequence dates the top of the overconsolidated section near the Stage 17/16 boundary $(\sim 670 \mathrm{ka})$.

\section{Carbon Isotopic Record}

The carbon isotopic record is remarkably stable during the interval from $\sim 620 \mathrm{ka}$ to the present, with the exception of large spikes of $\sim 0.5 \%$ o mainly during inferred interglacials (Fig. 4 ). Also, the top 20 $\mathrm{cm}$ features the highest $\delta^{13} \mathrm{C}$ values observed in the core $(-0.1 \%$ o to $0.4 \%$ ), suggesting a fundamentally different hydrography in the Holocene. The major features of the $\delta^{13} \mathrm{C}$ record, however, are two largeamplitude cycles of $\sim 1 \%$ within the overconsolidated section. A $1.3 \%$ increase coincides with the top of this section. Thus, the overconsolidated section is marked by a fundamentally different $\delta^{13} \mathrm{C}$ signal than the upper Quaternary section. Such large $\delta^{13} \mathrm{C}$ fluctuations are unknown in middle Quaternary global $\delta^{13} \mathrm{C}$ records (e.g., Shackleton and Hall, 1989; Berger et al., 1993). However, lower magnitude fluctuations are present in middle to late Quaternary benthic $\delta^{13} \mathrm{C}$ records from the North Atlantic (e.g., Ruddiman et al., 1989). The magnitude of Hole $910 \mathrm{~A} \delta^{13} \mathrm{C}$ cycles suggests highly variable conditions specific to the Yermak Plateau region prior to $\sim 620 \mathrm{ka}$. These $\delta^{13} \mathrm{C}$ fluctuations do not seem to correlate with any measured parameter of the Hole 910A sequence, including sedimentological parameters such as grain size, density, or porosity. Nevertheless, the $\sim 1 \%$ $\delta^{13} \mathrm{C}$ fluctuations prior to $\sim 620 \mathrm{ka}$ may well be genetically linked to the same processes that resulted in an overconsolidated section below $\sim 19$ mbsf.

Assuming that $N$. pachyderma $(\mathrm{s})$ reflects surface water $\delta^{13} \mathrm{C}$ values, the $\sim 1 \%$ variations prior to $\sim 620 \mathrm{ka}$ may reflect large changes in the $\delta^{13} \mathrm{C}$ of $\Sigma \mathrm{CO}_{2}$ over the Yermak Plateau. This assumption is based on recent findings that $N$. pachyderma (s) accurately records mixed layer $\delta^{13} \mathrm{C}$ in the Greenland Sea (Kohfeld et al., 1994; Johannessen et al., in press). Such changes might be produced either by variations in organic matter concentrations or by changes in "thermodynamic tagging" of surface water $\Sigma \mathrm{CO}_{2}$ by air-sea exchange (Charles and Fairbanks, 1990). Thermodynamic tagging should increase surface water $\delta^{13} \mathrm{C}$ (Charles and Fairbanks, 1990). However, modern surface waters at $80^{\circ} \mathrm{N}$ show significant thermodynamic depletion (Charles et al., 1993), yet Holocene $\delta^{13} \mathrm{C}$ values are the highest of the late Quaternary in Hole 910A. As the $\delta^{13} \mathrm{C}$ values within the overconsolidated section are anomalously low relative to modern values, the potential control by thermodynamic gas exchange seems unlikely.

Alternatively, large-scale changes in upwelling may explain the large $1 \% o \delta^{13} \mathrm{C}$ variations prior to $\sim 620 \mathrm{ka}$, as also observed in the central Arctic during the last deglaciation (Stein et al., 1994). Or, input of terrestrially derived organic carbon (with lower $\delta^{13} \mathrm{C}$ values) may have been greater, due to more dynamic glacial processes. In short, these large $\delta^{13} \mathrm{C}$ variations still require explanation, and the question is open as to how they relate to the processes of overconsolidation.

\section{Interpretations of the Overconsolidated Section}

Interpretations of the overconsolidated section include (1) episodic grounding of a marine-based ice sheet, perhaps derived from the Barents Sea ice sheet and buttressed by Svalbard, which overcompacted the sediments on the Yermak Plateau, and/or (2) coarsergrained glacial sedimentation that allowed enhanced compaction during this interval (Myhre, Thiede, Firth, et al., 1995; Rack et al., Chapter 21 , this volume). Overcompaction may have also resulted from large icebergs grounding at an inferred water depth of at least $400 \mathrm{~m}$. Grounding of recent icebergs on the Yermak Plateau at water depths of $\sim 450$ to $850 \mathrm{~m}$ has been demonstrated based on sidescan sonar and $3.5-\mathrm{kHz}$ profiling, although the age is not well known (Vogt et al., 1994). Also, the Yermak Plateau exhibits a beveled surface, which may indicate the presence of grounded ice during the late Quaternary (Vogt et al., 1994, 1995). In areas of ice sheet or iceberg grounding, large-scale erosion under the ice and redeposition are common (e.g., Vorren et al., 1988). A major hiatus indeed exists at the base of the 


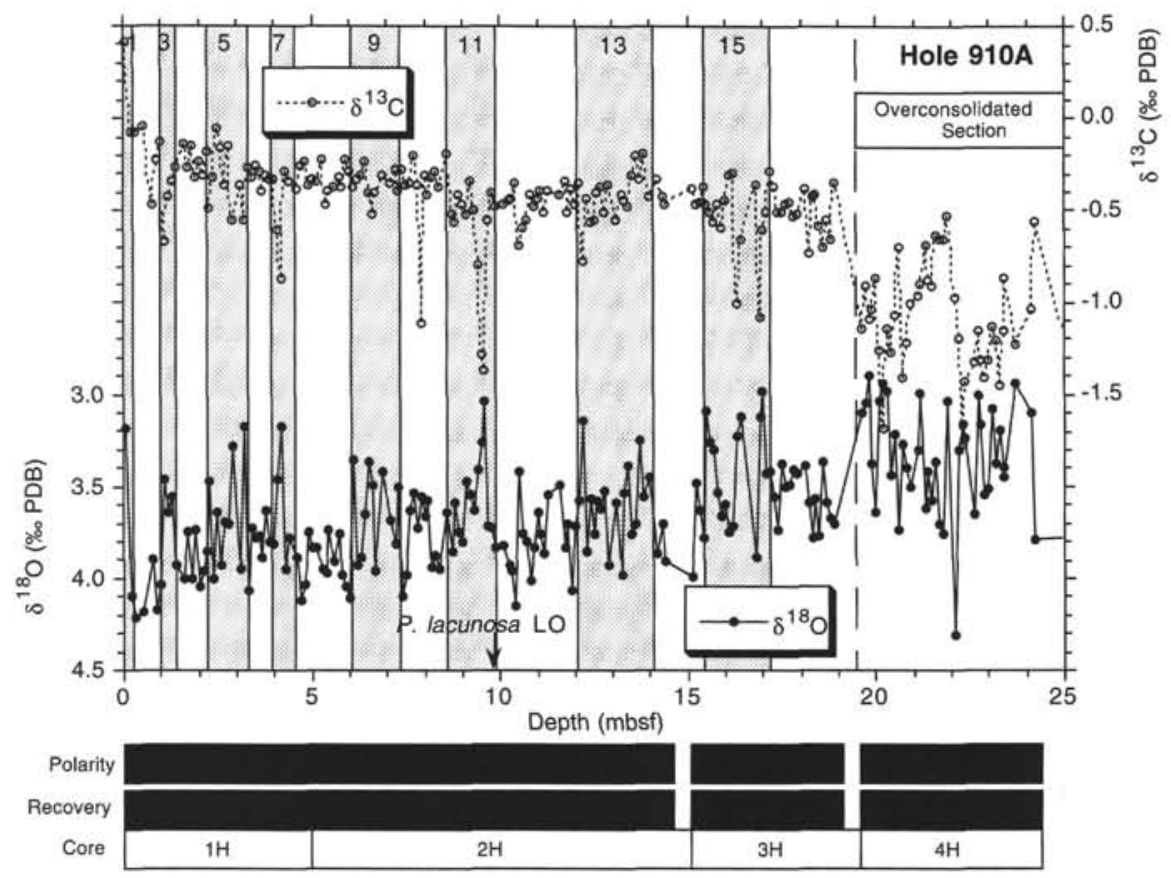

Figure 2. Hole $910 \mathrm{~A} \delta^{18} \mathrm{O}$ and $\delta^{13} \mathrm{C}$ data vs. depth, based on $N$. pachyderma (sinistrally coiling). Oxygen isotope Stages 1 through 16 are present in the upper 19 mbsf. The LO of P. lacunosa is at $9.7 \pm$ $0.75 \mathrm{mbsf}$. The overconsolidated section below $\sim 19.5$ mbsf is marked by large (>1\%o) shifts in $\delta^{13} \mathrm{C}$. Core recovery and normal magnetic polarity are shown in black. overconsolidated section between $\sim 70$ and $95 \mathrm{mbsf}$, but there is no evidence for a hiatus near the top at $\sim 19.5$ mbsf.

Alternatively, overcompaction may have resulted from coarser grained sedimentation, because sandy clays are more easily compacted than silty clays (Hamilton, 1976). There is some evidence for more numerous particles $>2 \mathrm{~mm}$ in diameter from $\sim 16$ to $25 \mathrm{mbsf}$, based on X-ray imaging of Hole 910D cores (Rack et al., Chapter 21, this volume). However, coarse grain size data show no mean differences in grain size ( $>63-$ and $>250-\mu \mathrm{m}$ fractions) in the overconsolidated section (Fig. 5), arguing against this hypothesis. Geotechnical and geomagnetic investigations are being conducted to further differentiate possible causes of overcompaction (Rack et al., Chapter 21, this volume; Solheim et al., unpubl. data; Williamson et al., unpubl. data).

\section{Quaternary Arctic Gateway Paleoceanography}

In addition to age control, stable isotopic data from Hole $910 \mathrm{~A}$ provide records of late Quaternary surface water environments in the Yermak Plateau region of the Arctic Ocean. The series of $\sim 1 \% \circ \delta^{18} \mathrm{O}$ cycles from 0 to $670 \mathrm{ka}$ are inferred to largely reflect the well-known variations in Quaternary ice volumes, although the record is complicated by minor salinity variations (Fig. 6). Salinity variations are significantly less than in the Arctic Ocean (e.g., Morris, 1988). Interestingly, the Hole $910 \mathrm{~A} \delta^{18} \mathrm{O}$ amplitude is $\sim 0.2 \%$ o to $0.4 \%$ o lower than the generally accepted $1.2 \% 0-1.4 \%$ attributed to Quaternary icegrowth and decay (e.g., Shackleton and Opdyke, 1973; Fairbanks and Matthews, 1978; Mix and Ruddiman, 1984; Fairbanks, 1989), although this may reflect minor undersampling of glacial/interglacial values. With this caveat, salinity effects appear to have been relatively minor, as the $\delta^{18} \mathrm{O}$ amplitude is not increased beyond the ice volume effect of $\sim 1.2 \%$ o to $1.4 \%$.

Possible explanations for dampened glacial-interglacial $\delta^{18} \mathrm{O}$ amplitudes on the Yermak Plateau relate to the regional oceanography. Surface water $\delta^{18} \mathrm{O}$ in this region is very sensitive to salinity differences (e.g., Fairbanks et al., 1992). The warm, high salinity WSC has a distinctly higher $\delta^{18} \mathrm{O}$ signature than the low salinity East Greenland Current (EGC) (by up to $1.5 \%$; Johannessen et al., in press). A higher influx of WSC waters during interglacials and a higher outflow of EGC waters during glacials would significantly dampen the glacial-interglacial $\delta^{18} \mathrm{O}$ amplitude. Alternatively, changes in the sea- sonal and/or depth preferences of $N$. pachyderma (s) may have resulted in dampened $\delta^{18} \mathrm{O}$ amplitudes.

In contrast to the last $670 \mathrm{k} . y ., \delta^{18} \mathrm{O}$ values prior to $\sim 670 \mathrm{ka}$ are $\sim 0.3 \%$ o lower, whereas maintaining a similar range of $\sim 1 \%$. This $\sim 0.3 \%$ difference is probably not attributable to lower global ice volumes of the middle Quaternary, based on the age control discussed above. Instead, the $\sim 0.3 \%$ difference may reflect a different temperature and/or salinity regime in the early Brunhes Chron. However, there is little evidence for warmer surface waters in the Norwegian Sea, which might have been advected into the Arctic Ocean via the West Spitsbergen Current and the Barents Sea branch. Indeed, the warmest temperatures in the Norwegian Sea are indicated for the entirety of the last $1 \mathrm{~m} . \mathrm{y}$. , based on planktonic foraminifer flux records and percentage carbonate records inferred to reflect temperature-dependent carbonate production (Jansen et al., 1988; Spiegler and Jansen, 1989; Henrich, 1989). Thus the $\sim 0.3 \%$ difference observed prior to $670 \mathrm{ka}$ may represent slightly lower mean salinities for Yermak Plateau surface waters (by $\sim 0.3 \%$, based on the calibration of Fairbanks et al., 1992).

Lower mean salinities might be due to (1) higher meltwater input and/or (2) a different glacial meltwater source marked by lower $\delta^{18} \mathrm{O}$. If the meltwater input were higher, the interval prior to $\sim 670 \mathrm{ka}$ might reflect greater instability in the circum-Arctic ice sheets. One would think, however, that the amplitude of the $\delta^{18} \mathrm{O}$ variations would be larger if such a mechanism were operating, but it was $\sim 1 \%$ both before and after $670 \mathrm{ka}$. Stated another way, both the glacial maxima and the interglacial minima are offset by $\sim 0.3 \%$, instead of preferential decreases during interglacials that might suggest stronger meltwater effects. Alternatively, surface water $\delta^{18} \mathrm{O}$ may have been influenced by a lower- $\delta^{18} \mathrm{O}$ glacial meltwater source. The $\delta^{18} \mathrm{O}$ of modern glacial ice masses in the Northern Hemisphere ranges from $\sim-10 \%$ o to $-30 \%$, dependent upon the moisture source and the altitude of deposition (e.g., Dansgaard et al., 1973). Thus, the $\sim 0.5 \%$ lower $\delta^{18} \mathrm{O}$ surface water values might reflect a different source, such as the larger Greenland ice sheet instead of the smaller Svalbard dome.

\section{Relation to the Middle Quaternary Transition}

The cause of the middle Quaternary climatic transition (from climates dominated by $\sim 41$ k.y. periodicity to $\sim 100$ k.y. periodicity, 
Table 2. Size fraction data from Hole 910A.

\begin{tabular}{|c|c|c|c|c|c|c|c|c|}
\hline $\begin{array}{l}\text { Core, section, } \\
\text { interval }(\mathrm{cm})\end{array}$ & $\begin{array}{l}\text { Depth } \\
\text { (mbsf) }\end{array}$ & $\begin{array}{l}\text { Age } \\
\text { (ka) }\end{array}$ & $\begin{array}{c}\text { Dry } \\
\text { weight }(\mathrm{g})\end{array}$ & $\begin{array}{c}>63-\mu \mathrm{m} \\
\text { weight }(\mathrm{g})\end{array}$ & $\begin{array}{l}>250-\mu \mathrm{m} \\
\text { weight }(\mathrm{g})\end{array}$ & $\begin{array}{l}\text { Core, section, } \\
\text { interval }(\mathrm{cm})\end{array}$ & $\begin{array}{l}\text { Depth } \\
\text { (mbsf) }\end{array}$ & $\begin{array}{l}\text { Age } \\
\text { (ka) }\end{array}$ \\
\hline 151-910A- & & & & & & $2 \mathrm{H}-3,110-112$ & 9.60 & 413.12 \\
\hline $1 \mathrm{H}-1,10-12$ & 0.10 & 9.23 & 12.7900 & 2.6933 & 0.8624 & $2 \mathrm{H}-3,120-122$ & 9.70 & 416.41 \\
\hline $1 \mathrm{H}-1,20-22$ & 0.20 & 14.70 & 13.5214 & 6.5308 & 3.6793 & $2 \mathrm{H}-3,130-132$ & 9.80 & 419.71 \\
\hline $1 \mathrm{H}-1,30-32$ & 0.30 & 18.55 & 8.8190 & 2.9000 & 0.8623 & $2 \mathrm{H}-3,140-142$ & 9.90 & 423.00 \\
\hline $1 \mathrm{H}-1,40-42$ & 0.40 & 22.40 & 18.2146 & 4.6014 & 0.5229 & $2 \mathrm{H}-4,10-12$ & 10.10 & 427.81 \\
\hline $1 \mathrm{H}-1,50-52$ & 0.50 & 26.259 & $\begin{array}{r}9.0720 \\
\end{array}$ & 4.6590 & 1.3875 & $2 \mathrm{H}-4,23-25$ & 10.23 & $\begin{array}{l}427.01 \\
430.94\end{array}$ \\
\hline $1 \mathrm{H}-1,60-62$ & 0.60 & 30.11 & 17.6799 & 11.4118 & 4.4616 & $2 \mathrm{H}-4,30-32$ & 10.30 & 432.62 \\
\hline $1 \mathrm{H}-1,70-72$ & 0.70 & 33.96 & 10.3050 & 2.3031 & 0.7382 & $2 \mathrm{H}-4,40-42$ & 10.40 & 435.02 \\
\hline $1 \mathrm{H}-1,80-82$ & 0.80 & 37.81 & 13.5770 & 2.7025 & 0.3359 & $2 \mathrm{H}-4,50-52$ & 10.50 & 437.43 \\
\hline $1 \mathrm{H}-1,90-92$ & 0.90 & 41.66 & 12.0370 & 2.2043 & 0.3447 & $2 \mathrm{H}-4,60-62$ & 10.60 & 439.83 \\
\hline $1 \mathrm{H}-1,100-102$ & 1.00 & 45.52 & 17.1058 & 3.6106 & 0.4480 & $2 \mathrm{H}-4,70-72$ & 10.70 & 442.24 \\
\hline $1 \mathrm{H}-1,110-112$ & 1.10 & 49.37 & 11.2560 & $\begin{array}{l}3.9127 \\
1.9127\end{array}$ & $\begin{array}{l}0.2342 \\
0.230\end{array}$ & $2 \mathrm{H}-4,82-84$ & 10.82 & $\begin{array}{l}442.24 \\
445.12\end{array}$ \\
\hline $1 \mathrm{H}-1,120-122$ & 1.20 & 53.22 & 13.8451 & 2.6222 & 0.3799 & $2 \mathrm{H}-4,90-92$ & 10.90 & 447.05 \\
\hline $1 \mathrm{H}-1,130-132$ & 1.30 & 57.07 & 13.4280 & 2.2468 & 0.3318 & $2 \mathrm{H}-4,100-102$ & 11.00 & 449.45 \\
\hline $1 \mathrm{H}-1,140-142$ & 1.40 & 60.82 & 14.2409 & 2.9141 & 0.4497 & $2 \mathrm{H}-4,110-112$ & 11.10 & 451.86 \\
\hline $1 \mathrm{H}-2,10-12$ & 1.60 & 68.08 & 10.6360 & 1.6720 & 0.2178 & $2 \mathrm{H}-4,120-122$ & 11.20 & 454.26 \\
\hline $1 \mathrm{H}-2,20-22$ & 1.70 & 71.71 & 13.9723 & 2.6230 & 0.3410 & $2 \mathrm{H}-4,130-132$ & 11.30 & 456.67 \\
\hline $1 \mathrm{H}-2,30-32$ & 1.80 & 75.34 & 15.2380 & 2.9195 & 0.7258 & $2 \mathrm{H}-5,10-12$ & 11.60 & 463.88 \\
\hline $1 \mathrm{H}-2,40-42$ & 1.90 & 78.97 & 14.1832 & 2.6779 & 0.3735 & $2 \mathrm{H}-5,23-25$ & 11.73 & 467.01 \\
\hline $1 \mathrm{H}-2,50-52$ & 2.00 & 82.61 & 12.9760 & 1.9912 & 0.3290 & $2 \mathrm{H}-5,30-32$ & 11.80 & 468.69 \\
\hline $1 \mathrm{H}-2,60-62$ & 2.10 & 86.24 & 15.9170 & 3.0524 & 0.4975 & $2 \mathrm{H}-5,40-42$ & 11.90 & 471.10 \\
\hline $1 \mathrm{H}-2,70-72$ & 2.20 & 89.87 & 15.4450 & 2.6566 & 0.3973 & $2 \mathrm{H}-5,50-52$ & 12.00 & 473.50 \\
\hline $1 \mathrm{H}-2,80-82$ & 2.30 & 93.50 & 14.4154 & 2.5932 & 0.3178 & $2 \mathrm{H}-5,60-62$ & 12.10 & 475.90 \\
\hline $1 \mathrm{H}-2,90-92$ & 2.40 & 97.13 & 12.9690 & 2.2477 & 0.3800 & $2 \mathrm{H}-5,70-72$ & 12.20 & 478.31 \\
\hline $1 \mathrm{H}-2,100-102$ & 2.50 & 100.76 & 17.5947 & 3.4031 & 0.4555 & $2 \mathrm{H}-5,80-82$ & 12.30 & 480.71 \\
\hline $1 \mathrm{H}-2,110-112$ & 2.60 & 104.39 & 11.7160 & 1.9300 & 0.2190 & $2 \mathrm{H}-5,90-92$ & 12.40 & 483.12 \\
\hline $1 \mathrm{H}-2,120-122$ & 2.70 & 108.03 & 14.4115 & 2.8332 & 0.4299 & $2 \mathrm{H}-5,100-102$ & 12.50 & 485.52 \\
\hline $1 \mathrm{H}-2,130-132$ & 2.80 & 111.66 & 10.6940 & 2.0075 & 0.4468 & $2 \mathrm{H}-5,110-112$ & 12.60 & 487.93 \\
\hline $1 \mathrm{H}-2,140-142$ & 2.90 & $\begin{array}{l}111.00 \\
11529\end{array}$ & $\begin{array}{l}15.6590 \\
15.6597\end{array}$ & 2.8086 & $\begin{array}{l}0.3746 \\
0.376\end{array}$ & $2 \mathrm{H}-5,120-122$ & 12.70 & $\begin{array}{l}487.93 \\
490.33\end{array}$ \\
\hline $1 \mathrm{H}-3,10-12$ & $\begin{array}{l}2.90 \\
3.10\end{array}$ & 122.55 & 17.3810 & $\begin{array}{l}2.8000 \\
3.3904\end{array}$ & 0. & $2 \mathrm{H}-5,130-132$ & 12.80 & 492.74 \\
\hline $1 \mathrm{H}-3,20-22$ & 3.20 & 126.18 & 16.9628 & 3.5216 & 0.6273 & $2 \mathrm{H}-5,140-142$ & 12.90 & 495.14 \\
\hline $1 \mathrm{H}-3,30-32$ & 3.30 & 132.33 & 13.7360 & 2.4487 & 0.4494 & $2 \mathrm{H}-6,10-12$ & 13.10 & $\begin{array}{l}490.14 \\
499.95\end{array}$ \\
\hline $1 \mathrm{H}-3,40-42$ & 3.40 & 141.00 & 16.9232 & 3.2938 & 0.3989 & $2 \mathrm{H}-6,23-25$ & 13.23 & 503.08 \\
\hline $1 \mathrm{H}-3,50-52$ & 3.50 & 149.67 & 13.4950 & 2.2338 & 0.2908 & $2 \mathrm{H}-6,30-32$ & 13.30 & 504.76 \\
\hline $1 \mathrm{H}-3,60-62$ & 3.60 & 158.33 & 16.0059 & 3.1865 & 0.47 & $2 \mathrm{H}-6,40-42$ & 13.40 & 507.17 \\
\hline $1 \mathrm{H}-3,70-72$ & $\begin{array}{l}3.00 \\
3.70\end{array}$ & 167.00 & $\begin{array}{l}10.0 \\
16.9\end{array}$ & $\begin{array}{l}3.1803 \\
2.7932\end{array}$ & 0. & $2 \mathrm{l}$ & 13.50 & 509.57 \\
\hline $1 \mathrm{H}-3,80-82$ & 3.80 & 175.67 & 19.4209 & 4.2777 & 1.0940 & $2 \mathrm{H}-6,60-62$ & 13.60 & 511.98 \\
\hline $1 \mathrm{H}-3,90-92$ & 3.90 & 184.33 & 14.0510 & 2.3665 & 0.3319 & $2 \mathrm{H}-6,70-72$ & 13.70 & 514.38 \\
\hline $1 \mathrm{H}-3,100-102$ & 4.00 & 193.00 & 18.5700 & 2.2843 & 0.2981 & $2 \mathrm{H}-6,80-82$ & 13.80 & 516.79 \\
\hline $1 \mathrm{H}-3,110-112$ & 4.10 & 201.67 & 15.5510 & $\begin{array}{l}2.2843 \\
1.9162\end{array}$ & $\begin{array}{l}0.2761 \\
0.2762\end{array}$ & $2 \mathrm{H}-6,90-92$ & 13.90 & 519.19 \\
\hline $1 \mathrm{H}-3,120-122$ & 4.20 & 210.33 & 17.6455 & 3.4332 & 0. & $2 \mathrm{H}-6,100-102$ & 14.00 & 521.60 \\
\hline $1 \mathrm{H}-3,130-132$ & 4.30 & 219.00 & 15.1950 & 2.7082 & $\begin{array}{l}0.4201 \\
0.5681\end{array}$ & $2 \mathrm{H}-\mathrm{CC}, 10-12$ & 14.19 & 526.83 \\
\hline $1 \mathrm{H}-3,140-142$ & 4.40 & 227.67 & 16.8316 & 3.3198 & 0.4429 & $2 \mathrm{H}-\mathrm{CC}, 23-25$ & 14.32 & 530.92 \\
\hline $1 \mathrm{H}-4,10-12$ & 4.60 & 245.00 & 15.8320 & 2.7449 & 0.4496 & $2 \mathrm{H}-\mathrm{CC}, 30-32$ & 14.39 & 533.13 \\
\hline $1 \mathrm{H}-4,20-22$ & 4.70 & 248.42 & 18.0760 & 3.5278 & 0.4403 & $3 \mathrm{H}-1,10-12$ & 15.20 & 558.62 \\
\hline $1 \mathrm{H}-4,30-32$ & 4.80 & 251.84 & 12.7760 & 2.3530 & 0.4 & $3 \mathrm{H}-1,20-22$ & 15.30 & 561.77 \\
\hline $1 \mathrm{H}-4,40-42$ & 4.90 & 255.25 & 15.3520 & 3.0033 & 0.5285 & $3 \mathrm{H}-1,30$ & 15.40 & 564.92 \\
\hline $1 \mathrm{H}-4,50-52$ & 5.00 & 258.67 & 16.8940 & 2.5923 & 0.3510 & $3 \mathrm{H}-1,40-42$ & 15.50 & 568.07 \\
\hline $1 \mathrm{H}-4,60-62$ & 5.10 & 262.09 & 20.9901 & 3.6791 & 0.4733 & $3 \mathrm{H}-1,50-52$ & 15.60 & 571.21 \\
\hline $1 \mathrm{H}-4,70-72$ & 5.20 & 265.51 & 18.8970 & 3.0069 & 0.4008 & $3 \mathrm{H}-1,60-62$ & 15.70 & 574.36 \\
\hline 1H-CC, $10-12$ & 5.36 & $\begin{array}{l}203.31 \\
270.98\end{array}$ & 17.6240 & 3.0 & 0.5 & $3 \mathrm{H}-1,70-72$ & 15.80 & 577.51 \\
\hline 1H-CC, $18-20$ & 5.44 & 273.71 & 18.8318 & 3.6484 & 0.5 & $3 \mathrm{H}-1,8$ & 15.91 & 580.97 \\
\hline $2 \mathrm{H}-1,10-12$ & 5.60 & 279.18 & 13.3511 & $\begin{array}{l}3.8534 \\
2.8532\end{array}$ & $\begin{array}{l}0.3415 \\
0.3688\end{array}$ & $\begin{array}{l}3 \mathrm{H}-1,81-83 \\
3 \mathrm{H}-1,90-92\end{array}$ & 16.00 & 583.80 \\
\hline $2 \mathrm{H}-1,23-25$ & 5.73 & 283.63 & 12.0385 & 2.3424 & 0.7213 & $3 \mathrm{H}-1,110-112$ & 16.10 & 586.95 \\
\hline $2 \mathrm{H}-1,30-32$ & 5.80 & 286.02 & 13.1117 & 2.1198 & 0.2439 & $3 \mathrm{H}-1,120-122$ & 16.20 & 590.10 \\
\hline $2 \mathrm{H}-1,40-42$ & 5.90 & 289.44 & 13.1545 & 2.5491 & 0.31 & $3 \mathrm{H}-1,133-135$ & 16.33 & 594.19 \\
\hline $2 \mathrm{H}-1,50-52$ & 6.00 & 292.85 & 10.9556 & 2.6 & 0.3 & $3 \mathrm{H}-1,140-142$ & 16.40 & 596.39 \\
\hline & $\begin{array}{l}0.00 \\
6.10\end{array}$ & $\begin{array}{l}292.85 \\
296.27\end{array}$ & 13.5544 & $\begin{array}{l}2.0612 \\
2.0656\end{array}$ & 0.2781 & $3 \mathrm{H}-2,10-12$ & 16 & 602 \\
\hline $2 \mathrm{H}-1,70-72$ & 6.20 & $\begin{array}{l}290.69 \\
299.69\end{array}$ & 14.2452 & $\begin{array}{l}2.0000 \\
2.4450\end{array}$ & 0.3275 & $3 \mathrm{H}-2,20-22$ & 16.70 & 605.84 \\
\hline $2 \mathrm{H}-1,80-82$ & 6. & 303.11 & 16.7326 & 3.2607 & 0.3 & $3 \mathrm{H}-2,3 \mathrm{C}$ & 16.80 & 608.98 \\
\hline $2 \mathrm{H}-1,90-92$ & 6. & 306.53 & 10.4790 & 1.9 & 0 & $3 \mathrm{H}$ & 16.90 & 13 \\
\hline $2 \mathrm{H}-1,100-102$ & & 309.95 & 1103 & 2.0 & 0.2 & 31 & 17.00 & 615.28 \\
\hline $2 \mathrm{H}-1,1 \mathrm{C}$ & $\begin{array}{l}0.50 \\
6.60\end{array}$ & $\begin{array}{l}309.95 \\
313.36\end{array}$ & $\begin{array}{r}11.032 \\
9492\end{array}$ & 2.0 & 0.2 & 31 & 17.10 & $\begin{array}{l}013.28 \\
618.43\end{array}$ \\
\hline $2 \mathrm{H}-1,120-122$ & $\begin{array}{l}0.00 \\
6.70\end{array}$ & $\begin{array}{l}313.30 \\
316.78\end{array}$ & $\begin{array}{r}9.4925 \\
12.9248\end{array}$ & $\begin{array}{l}2.03800 \\
2.5800\end{array}$ & $\begin{array}{l}0.4 \\
0.3\end{array}$ & $3 \mathrm{H}$ & 17.20 & $\begin{array}{l}010.43 \\
621.14\end{array}$ \\
\hline $1,140-142$ & 6.9 & 323.62 & 10.8 & & & 83 & 17.31 & 623.66 \\
\hline 2,10 & 7. & 330 & 10. & 2.0 & 0.2 & $3 \mathrm{H}$ & 17. & 72 \\
\hline 2,23 & 7. & 334 & 20.1 & 3.6 & & 102 & 17 & 01 \\
\hline $2 \mathrm{H}-2,30-32$ & 7.30 & 337.29 & $\begin{array}{l}20.19 \\
11.77\end{array}$ & 2.2. & & $3 \mathrm{H}-2$, & 17.60 & 63 \\
\hline $2 \mathrm{H}-2$, & 7.40 & 340.6 & 11.51 & 2.1 & 0.2 & $3 \mathrm{H}-2,120$ & 17. & 632 \\
\hline & 7.50 & 343.94 & 14.6505 & 2.8913 & 0.23 & $3 \mathrm{H}-2,132-134$ & 17. & \\
\hline 2 & 7.8 & 3. & 15.8 & & & -142 & 17. & 637.16 \\
\hline 70 & 7. & 350 & 19. & 3.6 & & 31 & 18 & 74 \\
\hline $2 \mathrm{H}-2,80$ & 7.80 & 353 & 17.3415 & & & $3 \mathrm{H}$ & 18.20 & 644.02 \\
\hline $2 \mathrm{H}-2,90-92$ & $\begin{array}{l}7.80 \\
7.90\end{array}$ & 357.12 & $\begin{array}{r}1,3415 \\
9.0922\end{array}$ & 1.7 & 0.2 .3 & 31 & $\begin{array}{l}18.20 \\
18.30\end{array}$ & 646.31 \\
\hline-102 & 8.00 & 360.41 & 12.4862 & 2.3 & & & $\begin{array}{l}10 \\
18\end{array}$ & 648 \\
\hline $\begin{array}{l}-112 \\
-112\end{array}$ & 8.0 & 41 & 11 & & & 31 & 18.48 & 650.43 \\
\hline 12 & & 3 & 11 & & & & $\begin{array}{l}18.40 \\
18.60\end{array}$ & \\
\hline $2 \mathrm{H}-2,130-132$ & 8. & 370.29 & 10.5095 & 2.0 & 0.29 & $3 \mathrm{H}-3,7 \mathrm{C}$ & 18.70 & \\
\hline $2 \mathrm{H}-2,140-142$ & 8.40 & 373.59 & 10.4627 & 20 & 0.31 & $3 \mathrm{H}-3,80-82$ & 18.80 & 657.75 \\
\hline $2 \mathrm{H}-3,10-12$ & $\begin{array}{l}8.40 \\
8.60\end{array}$ & 380. & $\begin{array}{l}10.46 \\
18.60\end{array}$ & $\begin{array}{l}2.0 \\
4.5\end{array}$ & & $3 \mathrm{H}-$ & $\begin{array}{l}18.80 \\
18.90\end{array}$ & 660.04 \\
\hline & $8.7 \quad-70$ & & & & & & $\begin{array}{l}19.60 \\
19.60\end{array}$ & 67 \\
\hline & & & & & & & 19.70 & \\
\hline $3,40-42$ & 8. & 390.06 & 17.5216 & 3.1812 & 0.4 & $4 \mathrm{H}-1.3$ & 19.80 & \\
\hline $2 \mathrm{H}-3,50-52$ & 9.00 & 393.35 & 13.9577 & 24016 & 0.3476 & $4 \mathrm{H}-1,40-42$ & 19.90 & 682.92 \\
\hline $2 \mathrm{H}-3,60-62$ & $\begin{array}{l}9.00 \\
9.10\end{array}$ & 396.65 & 12.2472 & $\begin{array}{l}2.40076 \\
2.6074\end{array}$ & 0.40 & $\begin{array}{l}4 \mathrm{H}-1,40-42 \\
4 \mathrm{H}-1,50-52\end{array}$ & 20.00 & 685.21 \\
\hline $2 \mathrm{H}-3,70-72$ & 9.20 & 399.9 & 16.0727 & 3.0 & 0.4 & $4 \mathrm{H}-1,60-62$ & 20.10 & 687.50 \\
\hline & 9.3 & 404. & & & & $4 \mathrm{H}-1,70-72$ & & 689.78 \\
\hline $2 \mathrm{H}-3,90$ & 9.4 & 406.53 & 12.3885 & 1.9880 & 0.2799 & $4 \mathrm{H}-1,80-82$ & 20.30 & 692.07 \\
\hline $2 \mathrm{H}-3,100-102$ & 9.50 & 409.82 & 11.4235 & 2.0021 & 0.3891 & $4 \mathrm{H}-1,90-93$ & 20.40 & 694.36 \\
\hline
\end{tabular}


Table 2 (continued).

\begin{tabular}{|c|c|c|c|c|c|}
\hline $\begin{array}{l}\text { Core, section, } \\
\text { interval }(\mathrm{cm})\end{array}$ & $\begin{array}{l}\text { Depth } \\
\text { (mbsf) }\end{array}$ & $\begin{array}{l}\text { Age } \\
\text { (ka) }\end{array}$ & $\begin{array}{c}\text { Dry } \\
\text { weight }(\mathrm{g})\end{array}$ & $\begin{array}{l}>63-\mu \mathrm{m} \\
\text { weight }(\mathrm{g})\end{array}$ & $\begin{array}{l}>250-\mu \mathrm{m} \\
\text { weight }(\mathrm{g})\end{array}$ \\
\hline $4 \mathrm{H}-1,100-102$ & 20.50 & 696.65 & 16.7926 & 3.3344 & 0.4933 \\
\hline $4 \mathrm{H}-1,110-112$ & 20.60 & 698.94 & 17.4143 & 3.5907 & 0.5054 \\
\hline $4 \mathrm{H}-1,120-122$ & 20.70 & 701.22 & 14.2512 & 2.4253 & 0.3654 \\
\hline $4 \mathrm{H}-1,130-132$ & 20.90 & 705.80 & 14.9513 & 4.3140 & 0.4861 \\
\hline $4 \mathrm{H}-1,140-142$ & 21.00 & 708.09 & 17.6662 & 3.1671 & 0.4861 \\
\hline $4 \mathrm{H}-2,10-12$ & 21.10 & 710.38 & 17.5194 & 4.2495 & 1.1751 \\
\hline $4 \mathrm{H}-2,18-20$ & 21.18 & 712.21 & 19.7941 & 3.7941 & 0.5023 \\
\hline $4 \mathrm{H}-2,30-32$ & 21.30 & 714.95 & 19.4657 & 3.8095 & 0.5628 \\
\hline $4 \mathrm{H}-2,40-42$ & 21.40 & 717.24 & 19.9762 & 3.9912 & 0.4233 \\
\hline $4 \mathrm{H}-2,50-52$ & 21.50 & 719.53 & 19.0619 & 3.8078 & 0.5319 \\
\hline $4 \mathrm{H}-2,60-62$ & 21.60 & 721.82 & 21.0652 & 3.7079 & 0.6981 \\
\hline $4 \mathrm{H}-2,70-72$ & 21.70 & 724.10 & 21.3747 & 3.8705 & 0.5191 \\
\hline $4 \mathrm{H}-2,80-82$ & 21.80 & 726.39 & 21.5116 & 2.9503 & 0.6118 \\
\hline $4 \mathrm{H}-2,90-92$ & 21.90 & 728.68 & 18.4486 & 3.6751 & 1.1586 \\
\hline $4 \mathrm{H}-2,100-102$ & 22.00 & 730.97 & 19.6751 & 14.1563 & 3.7986 \\
\hline $4 \mathrm{H}-2,110-112$ & 22.10 & 733.26 & 20.4067 & 3.9415 & 0.6113 \\
\hline $4 \mathrm{H}-2,120-122$ & 22.20 & 735.54 & 20.9667 & 3.7992 & 0.5625 \\
\hline $4 \mathrm{H}-2,130-132$ & 22.30 & 737.83 & 16.5172 & 2.8819 & 0.3797 \\
\hline $4 \mathrm{H}-2,138-140$ & 22.38 & 739.66 & 22.0873 & 3.9626 & 0.5913 \\
\hline $4 \mathrm{H}-3,10-12$ & 22.60 & 744.70 & 19.1143 & 3.6247 & 0.5140 \\
\hline $4 \mathrm{H}-3,20-22$ & 22,70 & 746.98 & 23.2025 & 4.6021 & 0.6094 \\
\hline $4 \mathrm{H}-3,30-32$ & 22.80 & 749.27 & 21.8700 & 4.8089 & 0.6799 \\
\hline $4 \mathrm{H}-3,40-42$ & 22.90 & 751.56 & 21.4349 & 4.5044 & 0.7586 \\
\hline $4 \mathrm{H}-3,50-52$ & 23.00 & 753.85 & 18.4450 & 3.0185 & 0.7903 \\
\hline $4 \mathrm{H}-3,60-62$ & 23.10 & 756.14 & 19.5414 & 3.7682 & 0.5150 \\
\hline $4 \mathrm{H}-3,70-72$ & 23.20 & 758.42 & 19.2720 & 3.8923 & 0.5620 \\
\hline $4 \mathrm{H}-3,80-82$ & 23.30 & 760.71 & 21.2068 & 3.9963 & 0.7920 \\
\hline $4 \mathrm{H}-3,90-92$ & 23.40 & 763.00 & 17.4301 & 3.5797 & 0.8838 \\
\hline $4 \mathrm{H}-3,100-102$ & 23.50 & 765.29 & 20.1906 & 3.5169 & 0.4924 \\
\hline $4 \mathrm{H}-3,110-112$ & 23.60 & 767.58 & 21.2460 & 4.0584 & 0.6693 \\
\hline $4 \mathrm{H}-3,120-122$ & 23.70 & 769.86 & 24.3649 & 4.9440 & 0.7899 \\
\hline $4 \mathrm{H}-3,130-132$ & 23.80 & 772.15 & 22.4093 & 11.1594 & 1.3096 \\
\hline $4 \mathrm{H}-4,10-12$ & 24.10 & 779.02 & 19.6622 & 3.7004 & 0.5208 \\
\hline $4 \mathrm{H}-4,20-22$ & 24.20 & 781.78 & 18.6604 & 3.1935 & 0.5446 \\
\hline
\end{tabular}

from $\sim 0.9$ to $0.6 \mathrm{Ma}$ ) is a central question in Quaternary paleoclimatology (e.g., Prell, 1982; Shackleton and Hall, 1989; Imbrie et al., 1993; Berger et al., 1993; Berger and Jansen, 1994). Marine shelf ice sheets (such as the Barents Sea ice sheet) are thought to play a major role in large-amplitude glacial-interglacial cycles of the late Quaternary (marked by $\sim 100$ k.y. periodicity), by allowing major ice buildup on marine shelves and rapid deglaciation (e.g., Broecker and van Donk, 1970; Shackleton and Opdyke, 1973; Vorren et al., 1988; Imbrie et al., 1993). Examination of circum-Arctic ice sheet history can help evaluate the possibility that larger, grounded ice sheets enhanced global $\delta^{18} \mathrm{O}$ amplitudes following the middle Quaternary climatic transition. The termination of the overconsolidated section near

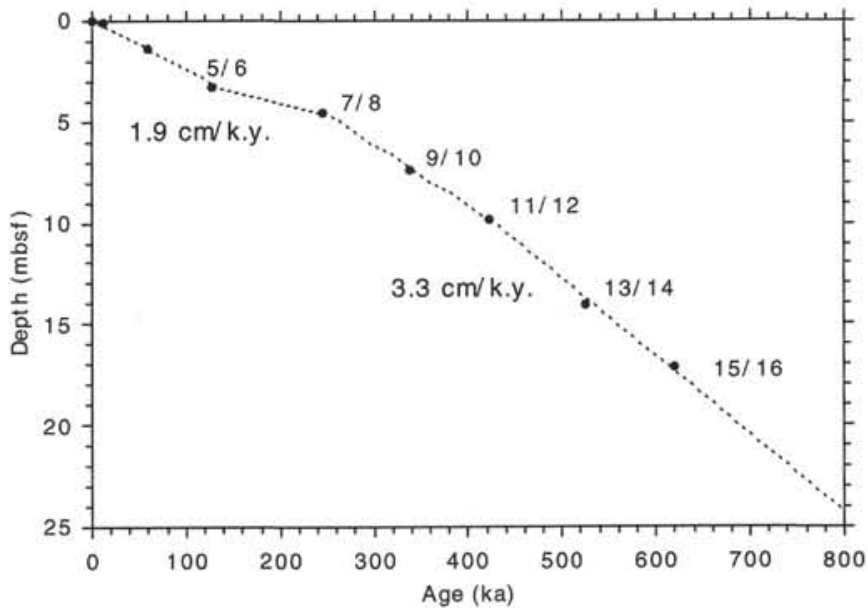

Figure 3. Age vs. depth plot of selected oxygen isotope stage boundaries used in an age model for Hole 910 A. Sedimentation rates average $\sim 1.9 \mathrm{~cm} / \mathrm{k} . \mathrm{y}$. for the $0-245 \mathrm{ka}$ interval and $~ 3.3 \mathrm{~cm} / \mathrm{k}$.y. for the $245-620 \mathrm{ka}$ interval.

$670 \mathrm{ka}$ coincides with the well-known increase in the climatic response to the $\sim 100$ k.y. period Milankovitch forcing (e.g., Berger et al., 1993). Conventional interpretation of the increased amplitude at the 100 k.y. period includes the supposition that grounded ice sheets became more common in the circum-Arctic during the late Quaternary (Hughes et al., 1977; Prell, 1982; Pollard, 1983; Vorren et al., 1988; Imbrie et al., 1993). Extensive ice buildup on the continental shelves of the Arctic Ocean during glacial intervals may have amplified the 100 k.y. climatic response and enhanced glacial-interglacial $\delta^{18} \mathrm{O}$ amplitudes. In this scenario, we might expect to find evidence for grounded marine-based ice sheets such as the Barents Sea ice sheet during glacial intervals after $\sim 800 \mathrm{ka}$ (starting during Stage 22, the first large late Quaternary glacial) and continuing during subsequent glacial intervals to oxygen isotope Stage 2. Evidence exists for several groundings of the Barents Sea ice sheet, probably within the last $800 \mathrm{ka}$ (Vorren et al., 1988), but the age is not well known.

Significantly, if the overconsolidated section represents grounding of a Svalbard/Barents Sea ice sheet, then this grounding was clearly most pervasive prior to $\sim 670 \mathrm{ka}$, and much less so toward the present. Thus, an age of $\sim 670 \mathrm{ka}$ for the top of this section may indi-

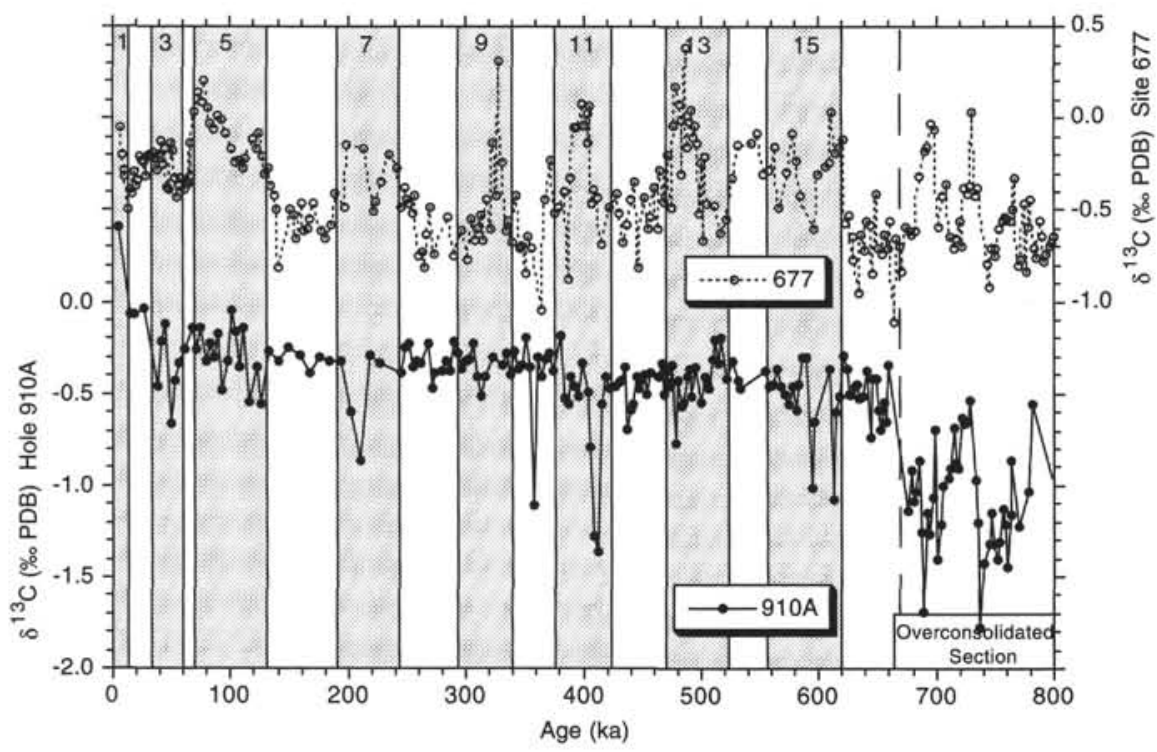

Figure 4. Carbon isotopic records for Hole 910A (based on $N$. pachyderma [sinistrally coiling]) and for Site 677 (based mainly on Uvigerina; Shackleton and Hall, 1989), plotted vs. age. Also shown are oxygen isotope stages. Note the general stability of Hole $910 \mathrm{~A} \delta^{13} \mathrm{C}$, except for large variations of $\sim 1 \%$ o prior to $\sim 670 \mathrm{ka}$. 
Figure 5. Hole 910A coarse fraction (wt \%) vs. age. No significant mean changes in percent $>63-\mu \mathrm{m}$ or percent $>250-\mu \mathrm{m}$ fractions are associated with the overconsolidated section. Also shown are oxygen isotope stages.

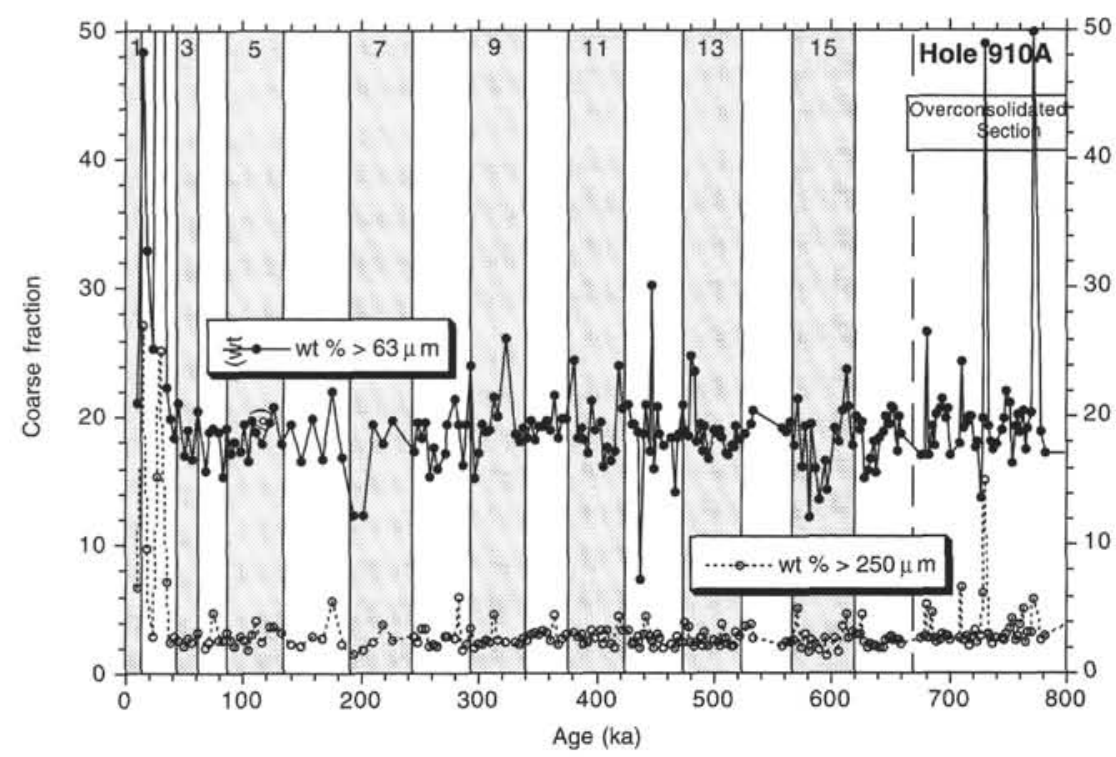

Figure 6. Oxygen isotopic records for Hole 910A (based on $N$. pachyderma [sinistrally coiling]) and for Site 677 (based mainly on Uvigerina; Shackleton and Hall, 1989), plotted vs. age. The top of the overconsolidated section at $\sim 19.5$ mbsf occurs near the Stage $17 / 16$ boundary $(670 \mathrm{ka})$. Also shown are oxygen isotope stages.

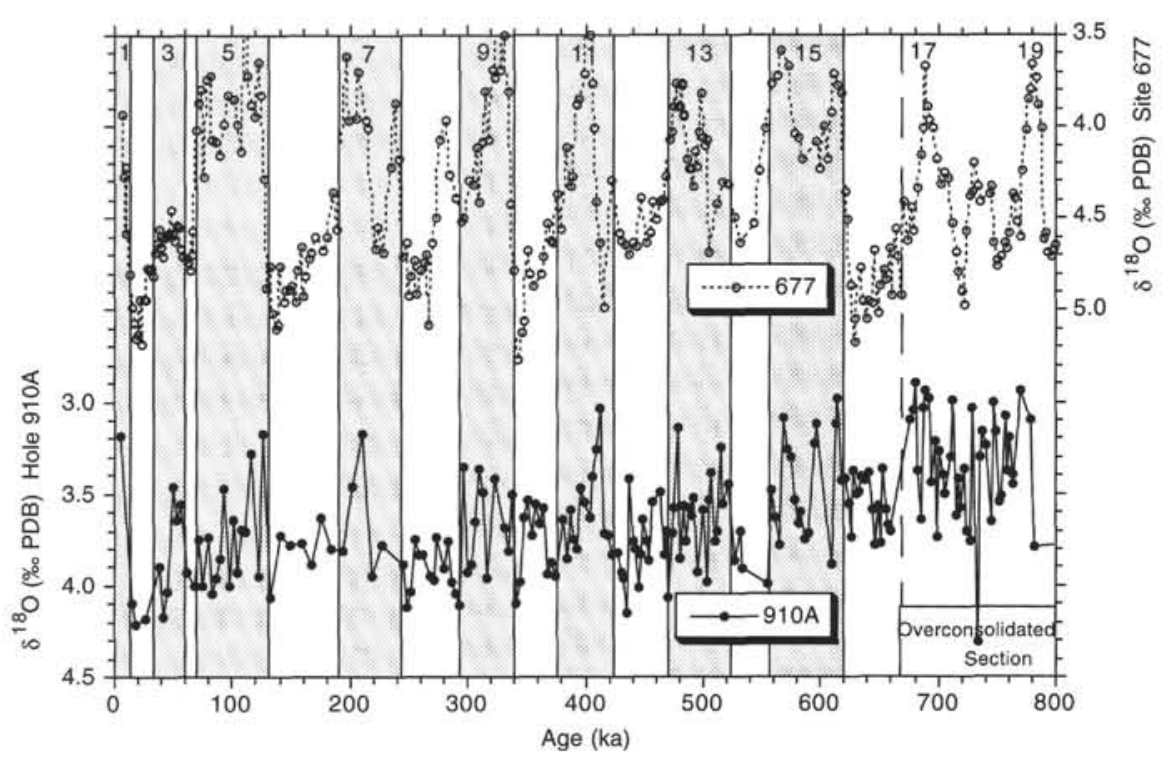

cate that grounded Svalbard/Barents Sea ice sheets did not become more common during the late Quaternary. One possible resolution is that the Barents Sea ice sheet may have undergone a transition from a grounded to a floating, marine-based ice sheet (e.g., Hughes et al., $1977)$ at this time. Such an ice sheet might still have produced the large glacial-interglacial variations in $\delta^{18} \mathrm{O}$ without overcompacting sediment on the Yermak Plateau.

Alternatively, the overconsolidated section may represent increased intensity of ice-rafting prior to $\sim 670 \mathrm{ka}$. Coarser grained glacial sedimentation might indicate greater ice-rafting through the Fram Strait, or possibly greater erosional capacity of the circum-Arctic ice sheets. The transition near $\sim 670$ ka may represent a change from very dynamic ice sheets (at least in the vicinity of the Yermak Plateau) to increased stability of the Arctic cryosphere, including, perhaps, the circum-Arctic ice sheets and the Arctic sea ice. Although the picture is not complete, the available evidence points to larger and/or more dynamic circum-Arctic ice sheets prior to $\sim 670 \mathrm{ka} \mathrm{com-}$ pared to after $\sim 670 \mathrm{ka}$. Whatever the cause of the overcompaction, the evidence, therefore, seems inconsistent with the idea that larger ice sheets became grounded more commonly in the late Quaternary.
Lastly, I speculate that the termination of ice-sheet grounding on the Yermak Plateau may correspond to a marked change in the character of Svalbard/Barents Sea glaciations during the middle Quaternary, and perhaps to the middle Quaternary climatic transition. Solheim et al. (in press) note a change from net erosion to net deposition on the western Svalbard Margin, which can be traced regionally as the Upper Regional Uncomformity (URU). This transition is attributed to "a shift from thick, eroding glaciers with steep ice profiles, to low profile fast flowing ice streams maintained by an increased amount of interglacial and interstadial sediments" (Solheim et al., in press). Detailed seismic profiling from the western Svalbard Margin to the Yermak Plateau (Solheim et al., unpubl. data) will test the potential link between the URU and the top of the overconsolidated section on the Yermak Plateau.

\section{SUMMARY}

Stable isotopic records from ODP Leg 151 Hole 910A based on N. pachyderma (s) represent some of the first high-resolution, long- 
term (middle Quaternary to present) records produced from the Arctic Ocean. The $\delta^{18} \mathrm{O}$ data reveal oxygen isotope Stages 1 through 16 (and possibly 17), although the record is complicated by salinity variations. Amplitudes of only $\sim 1 \%$ suggest salinity changes dampened the glacial-interglacial $\delta^{18} \mathrm{O}$ signal throughout the middle to late Quaternary. Termination of a remarkable overconsolidated section below $\sim 19.5 \mathrm{mbsf}$ is dated near the Stage $17 / 16$ boundary (=670 ka). Large $\delta^{13} \mathrm{C}$ fluctuations of $>1 \%$ o suggest significant changes in the $\delta^{13} \mathrm{C}$ of $\Sigma \mathrm{CO}_{2}$ prior to $\sim 670 \mathrm{ka}$. The large-amplitude variations in $\delta^{13} \mathrm{C}$ below $\sim 19.5 \mathrm{mbsf}$, and the overconsolidated nature of the section, suggest a fundamentally different sedimentary environment. Possible explanations include (1) episodic grounding of a marine-based ice sheet, perhaps derived from the Barents Sea ice sheet and buttressed by Svalbard, that overcompacted the sediment and strongly influenced surface water environments on the Yermak Plateau, and/or (2) coarser grained glacial sedimentation that allowed enhanced compaction during this interval. Whatever the cause of the overcompaction, its termination indicates a major change in Yermak Plateau sedimentation history near the Stage $17 / 16$ boundary $(670 \mathrm{ka})$. This termination may relate to a fundamental change in state of the Svalbard/Barents Sea ice sheets, and to the middle Quaternary climate transition. Further evaluation of late Quaternary circum-Arctic climate history is needed to better understand the role of the Arctic cryosphere in Quaternary climate change.

\section{ACKNOWLEDGMENTS}

This work was supported by the JOI/USSSP (151-20794b), along with partial support from NSF (OPP-9423485). I thank the Ocean Drilling Program for inviting my participation on Leg 151; Nao Ahagon for providing samples; D.W. Oppo, W.F. Ruddiman and an anonymous reviewer for helpful reviews; and Melany Manaig, Bhavna Sahi, and Diana Taylor for laboratory assistance.

\section{REFERENCES}

Berger, W.H., Bickert, T., Schmidt, H., and Wefer, G., 1993. Quaternary oxygen isotope record of pelagic foraminifers: Site 806, Ontong Java Plateau. In Berger, W.H., Kroenke, L.W., Mayer, L.A., et al., Proc. ODP, Sci. Results, 130: College Station, TX (Ocean Drilling Program), 381-395.

Berger, W.H., and Jansen, E., 1994. Mid-Pleistocene climate shift: the Nansen connection. In Johannessen, O.M., Muensch, R.D., and Overland, J.E. (Eds.), The Role of the Polar Oceans in Shaping the Global Environment. Geophys. Monogr., Am. Geophys. Union, 85:295-311.

Broecker, W.S., and van Donk, J., 1970. Insolation changes, ice volumes, and the $\mathrm{O}^{18}$ record in deep-sea cores. Rev. Geophys. Space Phys., 8:169-198.

Charles, C.D., and Fairbanks, R.G., 1990. Glacial to interglacial changes in the isotopic gradients of Southern Ocean surface water. In Bleil, U., and Thiede, J. (Eds.), Geological History of Polar Oceans: Arctic Versus Antarctic: Netherlands (Kluwer Academic), 519-538.

Charles C.D., Wright, J.D., and Fairbanks, R.G., 1993. Thermodynamic influences on the marine carbon isotope record. Paleoceanography, 8:691-698.

Dansgaard, W., Johnsen, S.J., Clausen, H.B., and Gundestrup, N., 1973. Stable isotope glaciology. Medd. Groenl., 197:1-53.

Fairbanks, R.G., 1989. A 17,000-year glacio-eustatic sea level record: influence of glacial melting rates on the Younger Dryas event and deep-ocean circulation. Nature, 342:637-642.

Fairbanks, R.G., Charles, C.D., and Wright, J.D., 1992. Origin of global meltwater pulses. In Taylor, R.E., Long, A., and Kra, R.S. (Eds.), Radiocarbon After Four Decades: New York (Springer-Verlag).

Fairbanks, R.G., and Matthews, R.K., 1978. The marine oxygen isotope record in Pleistocene coral, Barbados, West Indies. Quat. Res., 10:181196.

Hamilton, E.L., 1976. Variations of density and porosity with depth in deepsea sediments. J. Sediment. Petrol., 46:280-300.

Henrich, R., 1989. Glacial/interglacial cycles in the Norwegian Sea: sedimentology, paleoceanography, and evolution of late Pliocene to Quaternary Northern Hemisphere climate. In Eldholm, O., Thiede, J., Taylor, E., et al., Proc. ODP, Sci. Results, 104: College Station, TX (Ocean Drilling Program), 189-232.

Hughes, T.C., Denton, G.H., and Grosswald, M.G., 1977. Was there a late Würm Arctic Ice Sheet? Nature, 266:596-602.

Imbrie, J., Berger, A., Boyle, E., Clemens, S., Duffy, A., Howard, W., Kukla, G., Kutzbach, J., Martinson, D., McIntyre, A., Mix, A., Molfino, B., Morley, J., Peterson, L., Pisias, N., Prell, W., Raymo, M., Shackleton, N., and Toggweiler, J., 1993. On the structure and origin of major glaciation cycles, 2 . The 100,000-year cycle. Paleoceanography, 8:699-735.

Imbrie, J., Hays, J.D., Martinson, D.G., McIntyre, A., Mix, A.C., Morley, J.J., Pisias, N.G., Prell, W.L., and Shackleton, N.J., 1984. The orbital theory of Pleistocene climate: support from a revised chronology of the marine $\delta^{18} \mathrm{O}$ record. In Berger, A., Imbrie, J., Hays, J., Kukla, G., and Saltzman, B. (Eds.), Milankovitch and Climate (Pt. 1), NATO ASI Ser. C, Math Phys. Sci., 126: Dordrecht (D. Reidel), 269-305.

Jansen, E., Bleil, U., Henrich, R., Kringstad, L., and Slettemark, B., 1988. Paleoenvironmental changes in the Norwegian Sea and Northeast Atlantic during the last 2.8 m.y.: Deep Sea Drilling Project/Ocean Drilling Program Sites 610, 642, 643, and 644. Paleoceanography, 3:563-581.

Johannessen, T., Ravelo, A.C., and Jansen, E., in press. Distribution of carbon and oxygen isotopes in the Greenland, Iceland and Norwegian seas: relationship to water masses, nutrients and circulation. Mar. Geol.

Kohfeld, K.E., Fairbanks, R.G., Smith, S.L., and Walsh, I.D., 1994. Decoupling of abundances and geochemical signals of $N$. pachyderma (s.) in marine sediments: evidence from MOCNESS plankton tows. Eos, $75: 392$.

Leg 151 Shipboard Scientific Party, 1994. Farthest north: Ocean drilling in the Arctic Gateway region. GSA Today, 5:24-33.

Mix, A.C., and Ruddiman, W.F., 1984. Oxygen-isotope analyses and Pleistocene ice volumes. Quat. Res., 21:1-20.

Morris, T.H., 1988. Stable isotope stratigraphy of the Arctic Ocean: Fram Strait to central Arctic. Palaeogeogr., Palaeoclimatol., Palaeoecol., 64:201-219.

Myhre, A.M., Thiede, J., Firth, J.V., et al., 1995. Proc. ODP, Init. Repts., 151: College Station, TX (Ocean Drilling Program).

Niitsuma, N., Oba, T., and Okada, M., 1991. Oxygen and carbon isotope stratigraphy at Site 723, Oman Margin. In Prell, W.L., Niitsuma, N., et al., Proc. ODP, Sci. Results, 117: College Station, TX (Ocean Drilling Program), 321-341.

Pollard, D., 1983. A coupled climate-ice sheet model applied to the Quaternary ice ages. J. Climatol., 1:965-997.

Prell, W.L., 1982. Oxygen and carbon isotope stratigraphy for the Quaternary of Hole 502B: evidence for two modes of isotopic variability. In Prell, W.L., Gardner, J.V., et al., Init. Repts. DSDP, 68: Washington (U.S. Govt. Printing Office), 455-464.

Raffi, I., Backman, J., Rio, D., and Shackleton, N.J., 1993. Plio-Pleistocene nannofossil biostratigraphy and calibration to oxygen isotopes stratigraphies from Deep Sea Drilling Project Site 607 and Ocean Drilling Program Site 677. Paleoceanography, 8:387-408.

Ruddiman, W.F., Raymo, M.E., Martinson, D.G., Clement, B.M., and Backman, J., 1989. Pleistocene evolution: Northern Hemisphere ice sheets and North Atlantic Ocean. Paleoceanography, 4:353-412.

Shackleton, N.J., Berger, A., and Peltier, W.A., 1990. An alternative astronomical calibration of the lower Pleistocene timescale based on ODP Site 677. Trans. R. Soc. Edinburgh: Earth Sci., 81:251-261.

Shackleton, N.J., and Hall, M.A., 1989. Stable isotope history of the Pleistocene at ODP Site 677. In Becker, K., Sakai, H., et al., Proc. ODP, Sci. Results, 111: College Station, TX (Ocean Drilling Program), 295-316.

Shackleton, N.J., and Opdyke, N.D., 1973. Oxygen isotope and paleomagnetic stratigraphy of equatorial Pacific core V28-238: oxygen isotope temperatures and ice volumes on a $10^{5}$ year and $10^{6}$ year scale. Quat. Res., 3:39-55.

Shipboard Scientific Party, 1995. Site 910. In Myhre, A.M., Thiede, J., Firth, J.V., et al., Proc. ODP, Init. Repts., 151: College Station, TX (Ocean Drilling Program), 221-270.

Solheim, A., Anderson, E.S., Elverhøi, A., and Fiedler, A., in press. Late Cenozoic depositional history of the western Svalbard continental shelf, controlled by subsidence and climate. Global Planet. Change.

Spiegler, D., and Jansen, E., 1989. Planktonic foraminifer biostratigraphy of Norwegian Sea sediments: ODP Leg 104. In Eldholm, O., Thiede, J., Taylor, E., et al., Proc. ODP, Sci. Results, 104: College Station, TX (Ocean Drilling Program), 681-696.

Stein, R., Schubert, C., Vogt, C., and Futterer, D., 1994. Stable isotope stratigraphy, sedimentation rates, and salinity changes in the latest Pleis- 
tocene to Holocene eastern central Arctic Ocean. Mar. Geol., 119:333355.

Thierstein, H.R., Geitzenauer, K., Molfino, B., and Shackleton, N.J., 1977. Global synchroneity of late Quaternary coccolith datum levels: validation by oxygen isotopes. Geology, 5:400-404.

Vogt, P.R., Crane, K., and Sudor, E., 1994. Deep Pleistocene iceberg plowmarks on the Yermak Plateau: sidescan and $3.5 \mathrm{kHz}$ evidence for thick calving ice fronts and a possible marine ice sheet in the Arctic Ocean. Geology, 22:403-406.

, 1995. Reply to Comment on "Deep Pleistocene iceberg plowmarks on the Yermak Plateau: sidescan and $3.5 \mathrm{kHz}$ evidence for thick calving ice fronts and a possible marine ice sheet in the Arctic Ocean." Geology, 23:477-478.

Vorren, T.O., Hald, M., and Legesbye, E., 1988. Late Cenozoic environments in the Barents Sea. Paleoceanography, 3: 601-612.

Date of initial receipt: 7 July 1995

Date of acceptance: 1 December 1995

Ms 151SR-133 